\title{
A modulation model for the solar and lunar daily geomagnetic variations
}

\author{
Frans De Meyer \\ Royal Meteorological Institute, Ringlaan 3, B-1180 Brussels, Belgium
}

(Received December 9, 2002; Revised June 24, 2003; Accepted July 25, 2003)

\begin{abstract}
The traditional Chapman-Miller analysis for the solar, $\mathbf{S}$, and lunar, $\mathbf{L}$, geomagnetic variations is generalized by incorporating a time description of the seasonal changes of the harmonic coefficients. The modulation model consists of the sum of harmonic oscillators with basic carriers having the fundamental frequencies of the solar and lunar daily components, which are being amplitude and phase modulated by the annual variation and its harmonics. The solar cycle effect is a priori taken into account by using the daily sunspot numbers as an auxiliary input and including the Wolf ratios in the amplitude and phase terms. For the station Dourbes (Belgium), solar and lunar harmonics show a marked increase in amplitude from winter to summer, but the seasonal changes of $\mathbf{L}$ significantly exceed that of $\mathbf{S}$. The phase shift from winter to summer in $\mathbf{L}$ is about three times that of $\mathbf{S}$. The Wolf ratios of the Fourier amplitudes are of the same order of magnitude for both the $\mathbf{S}$ and $\mathbf{L}$ variations. Removal of the relatively important ocean dynamo contribution does not have an appreciable consequence for the determination of the seasonal changes and the sunspot cycle influence.
\end{abstract}

Key words: Solar and lunar daily magnetic variations, Chapman-Miller method, modulation model.

\section{Introduction}

The geomagnetic field measured at any point on the Earth as a function of time shows periodic variations due to atmospheric processes caused by the Sun and the Moon. Both $\mathbf{S}$ and $\mathbf{L}$, respectively the solar and lunar daily geomagnetic variations, are commonly assumed to result mainly from electric currents generated by dynamo action in the ionosphere, which is generally a much better conductor during the day than during the night, except in the auroral regions. This dynamo is powered by tidal motions of the conductive layers of the ionosphere across lines of force of the Earth's magnetic field, the tides being of mainly thermal origin of $\mathbf{S}$ and of purely gravitational origin of $\mathbf{L}$. The currents flow partly in the ionosphere, partly along lines of force, and also within the Earth itself as a result of induction. Moreover, the observed $\mathbf{L}$ variations contain a contribution from an ocean dynamo (Malin, 1970).

Both the solar and lunar geomagnetic daily variations have been known to vary with season. The seasonal changes of $\mathbf{S}$, particularly in its quiet day form, $\mathbf{S}_{\mathrm{q}}$, have been studied in much detail, but the seasonal changes of $\mathbf{L}$ are less well known. Of particular interest is the contrast between the seasonal variations of $\mathbf{S}$ and $\mathbf{L}$. It is also desirable to compare the changes of $\mathbf{S}$ and $\mathbf{L}$ with the solar cycle. That the sunspot cycle influences $\mathbf{S}$ has never been in doubt, but there is some incertitude over the response of $\mathbf{L}$ to the solar cycle.

A high-resolution spectral analysis of the geomagnetic data shows the splitting of the solar and lunar lines by the annual variation and its harmonics. The minor spectral peaks are attributed to a $\sim 27$-day amplitude modulation of the solar diurnal line $\mathbf{S}_{1}$ (Black, 1970). The Chapman-Miller

Copy right (c) The Society of Geomagnetism and Earth, Planetary and Space Sciences (SGEPSS); The Seismological Society of Japan; The Volcanological Society of Japan; The Geodetic Society of Japan; The Japanese Society for Planetary Sciences.
(1940) method is generally used for the determination of the first four harmonics of the solar and lunar variations in the geomagnetic field. Important cyclic and seasonal changes are present in the Fourier amplitudes and phases (De Meyer, 1986).

It is possible to interpret the main spectral peaks in the diurnal band and its harmonics in terms of amplitude and phase modulation of the solar and lunar lines. The associated modulation model is composed of the sum of harmonic oscillators, with basic carriers having the fundamental frequencies of $\mathbf{S}$ and $\mathbf{L}$, respectively, which are being amplitude and phase modulated by long-term variations that can be described by a sum of trigonometric terms. The solar cycle influence is modelled by incorporating the effect of the daily sunspot numbers a priori in the amplitude and phase terms, thereby simulating the high degree of day-to-day variation in the harmonic coefficients.

The data used in this analysis consist of the hourly mean values of magnetic declination (D-component), measured at the station of Dourbes, Belgium $\left(50^{\circ} 06^{\prime} \mathrm{N}, 4^{\circ} 36^{\prime} \mathrm{E}\right)$ for the interval January 1, 1960 to December 31,1999 . The values of $\mathrm{D}$ are given in minutes of arc and the first value of each day corresponds to 00.30 UT. At the dip latitude of Dourbes $\left(51.7^{\circ} \mathrm{N}\right)$, periodic variations in the frequency interval 0 to 4 cycles per day are most conspicuous in the declination, so that this magnetic element was chosen for the investigation.

\section{Data and Spectral Analysis}

With a view to analyse the frequency structure of the main solar frequency bands separately, the following data processing is performed on the original record of $N=350640$ hourly readings. The power around the solar lines $\mathbf{S}_{n}, n=$ $1,2,3,4$, is isolated by applying four different band-pass filters, each of total bandwidth 0.03 cycles/hour, having the 


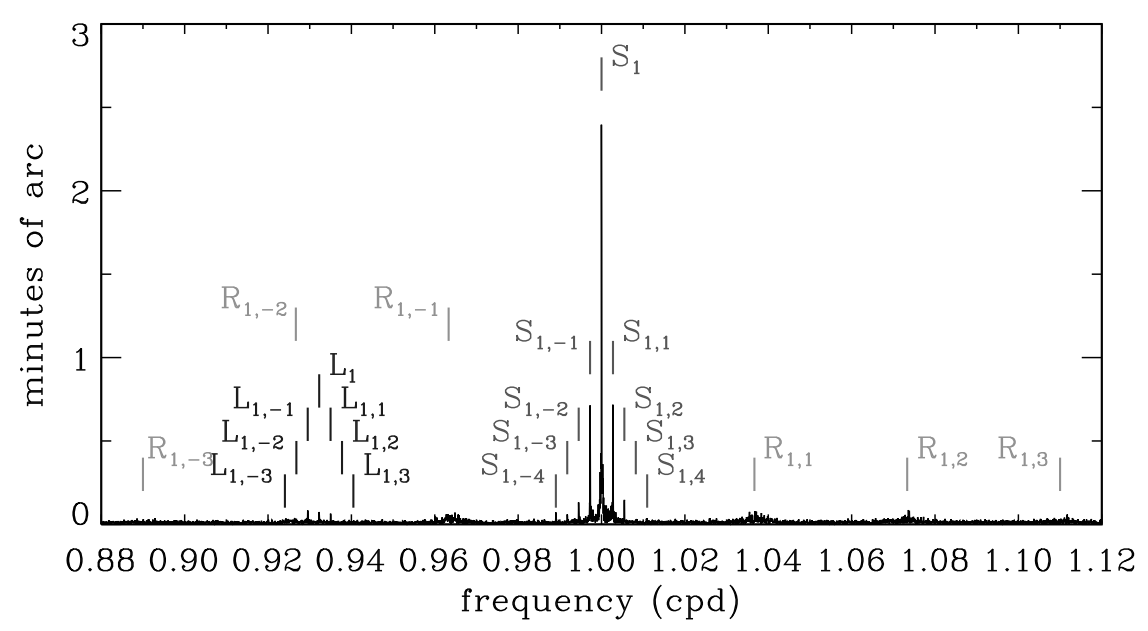

Fig. 1. Amplitude spectrum of the declination in the diurnal band.

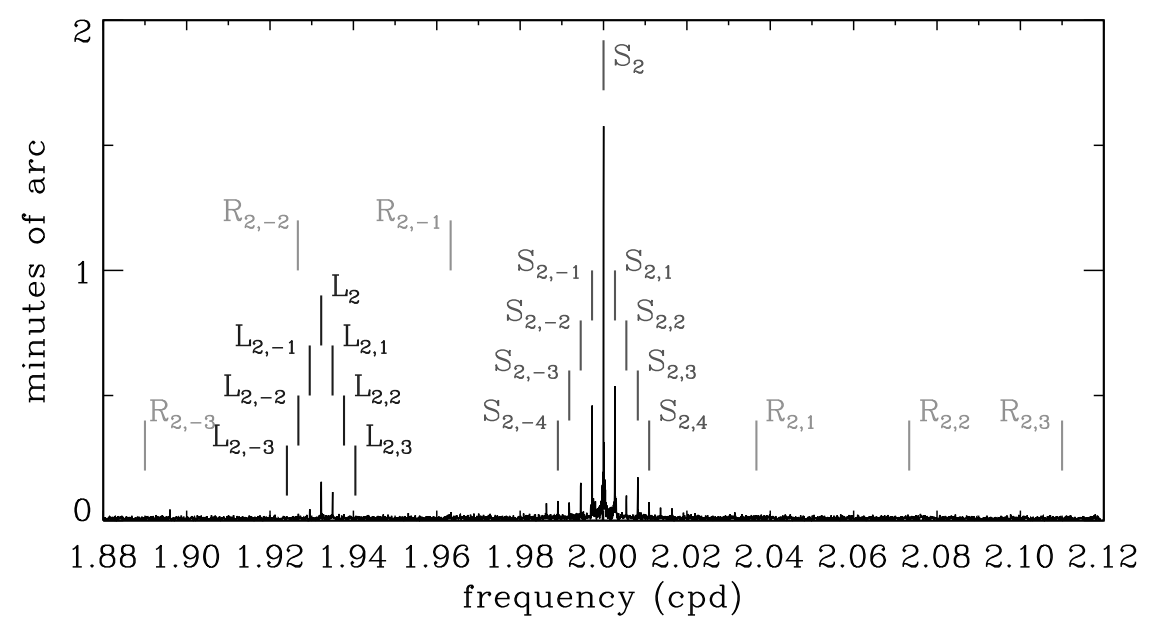

Fig. 2. Amplitude spectrum of the declination in the semi-diurnal band.

property that the four resulting output sequences only contain the respective contributions of the input signal to the solar diurnal band and its main harmonics. The specific bandpass filter for the $n$th harmonic consists of 200 symmetrical impulse response weights and removes the constituents at the frequencies outside the tidal wave band (Winch and Cunningham, 1972) of the solar daily harmonic of frequency $n$ cycles per mean solar day. The lunar variations are evidently maintained in the output series by this numerical procedure. The frequency responses of these band-pass filters are evidently taken into account when the amplitudes of the magnetic variations are determined. No phase shift is introduced by the symmetrical band-pass filtering.

Next, the four sub-sequences are summed and the resulting output is sampled by keeping every second point only, thus obtaining a time series of $N / 2=175321$ bi-hourly data. Since observations are lost at the beginning and the end of the input record as a consequence of the filtering process, we end up with 175121 data which are restricted to the frequency range $(0,6)$ cycles/day (cpd). As a result, the high-frequency noise and the contributions outside the solar bands are severely attenuated. It is important to note that this band-pass filtering scheme acts as a trend-removal technique in the sense that the long-term trend, originating from the secular variation, is practically nullified.

To determine the fine structure of the solar frequency bands, a high resolution spectrum was computed using the Fast Fourier transform (FFT). Restricting the outcomes of the spectral analysis to the diurnal and semi-diurnal frequency bands, Figures 1 and 2 show sections of the amplitude spectrum around 1 and $2 \mathrm{cpd}$ for the filtered declination data. The spectral peaks are conspicuously resolved because of the very extended horizontal scale as we have used a frequency resolution of $3.4210^{-5} \mathrm{cpd}$. Analysis at this resolution splits the solar diurnal and semi-diurnal peaks into lines $\mathbf{S}_{1}$ and $\mathbf{S}_{2}$ and sidebands at the frequencies $(n+k / 365.25)$ cpd, $n=1,2 ; k= \pm 1, \pm 2, \ldots$, originating from the modulation of the solar daily variation by the annual fluctuation and its harmonics. Sidebands of $\mathbf{S}_{2}$ up to the sixth order are seen in Fig. 2. In general, these sidebands are denoted by $\mathbf{S}_{n, k}, n=1,2, \ldots ; k= \pm 1, \pm 2, \ldots$ (Chapman and Malin, 1970).

The investigation of small periodic variations with frequencies in the neighbourhood of the lunar lines is particularly enigmatic as it involves the identification of weak lines, clustered in the vicinity of the lunar waves $\mathbf{L}_{1}$ and $\mathbf{L}_{2}$ and yet in the presence of relatively strong noise in the solar bands. The two dominating lines in Fig. 2 for the semi-diurnal band 
Table 1. Declination-Chapman-Miller harmonic analysis.

\begin{tabular}{|c|c|c|c|c|c|c|c|c|}
\hline & \multicolumn{4}{|c|}{ Solar term $\mathbf{S}_{n}$} & \multicolumn{4}{|c|}{ Lunar terms $\mathbf{L}_{n}$} \\
\hline & $n$ & $s_{n}$ & $r_{n}$ & $\sigma_{n}$ & $n$ & $\ell_{n}$ & $\rho_{n}$ & $\lambda_{n}$ \\
\hline \multirow[t]{4}{*}{ All data } & 1 & $2.584^{\prime}$ & $0.011^{\prime}$ & $57.2^{\circ}$ & 1 & $0.086^{\prime}$ & $0.012^{\prime}$ & $148.8^{\circ}$ \\
\hline & 2 & $1.767^{\prime}$ & $0.006^{\prime}$ & $230.6^{\circ}$ & 2 & $0.168^{\prime}$ & $0.006^{\prime}$ & $262.6^{\circ}$ \\
\hline & 3 & $0.789^{\prime}$ & $0.004^{\prime}$ & $79.5^{\circ}$ & 3 & $0.068^{\prime}$ & $0.005^{\prime}$ & $128.5^{\circ}$ \\
\hline & 4 & $0.270^{\prime}$ & $0.003^{\prime}$ & $273.8^{\circ}$ & 4 & $0.012^{\prime}$ & $0.003^{\prime}$ & $310.7^{\circ}$ \\
\hline \multirow[t]{4}{*}{$j$-months } & 1 & $3.551^{\prime}$ & $0.015^{\prime}$ & $42.9^{\circ}$ & 1 & $0.199^{\prime}$ & $0.017^{\prime}$ & $120.7^{\circ}$ \\
\hline & 2 & $2.413^{\prime}$ & $0.009^{\prime}$ & $244.6^{\circ}$ & 2 & $0.302^{\prime}$ & $0.010^{\prime}$ & $275.1^{\circ}$ \\
\hline & 3 & $0.866^{\prime}$ & $0.008^{\prime}$ & $87.7^{\circ}$ & 3 & $0.120^{\prime}$ & $0.008^{\prime}$ & $122.5^{\circ}$ \\
\hline & 4 & $0.038^{\prime}$ & $0.006^{\prime}$ & $248.4^{\circ}$ & 4 & $0.006^{\prime}$ & $0.006^{\prime}$ & $223.5^{\circ}$ \\
\hline \multirow[t]{4}{*}{$e$-months } & 1 & $2.768^{\prime}$ & $0.014^{\prime}$ & $60.2^{\circ}$ & 1 & $0.113^{\prime}$ & $0.015^{\prime}$ & $159.3^{\circ}$ \\
\hline & 2 & $1.987^{\prime}$ & $0.011^{\prime}$ & $229.6^{\circ}$ & 2 & $0.166^{\prime}$ & $0.012^{\prime}$ & $262.0^{\circ}$ \\
\hline & 3 & $1.083^{\prime}$ & $0.009^{\prime}$ & $73.0^{\circ}$ & 3 & $0.082^{\prime}$ & $0.009^{\prime}$ & $134.8^{\circ}$ \\
\hline & 4 & $0.448^{\prime}$ & $0.007^{\prime}$ & $278.7^{\circ}$ & 4 & $0.035^{\prime}$ & $0.007^{\prime}$ & $327.7^{\circ}$ \\
\hline \multirow[t]{4}{*}{$d$-months } & 1 & $1.697^{\prime}$ & $0.015^{\prime}$ & $83.3^{\circ}$ & 1 & $0.085^{\prime}$ & $0.017^{\prime}$ & $261.4^{\circ}$ \\
\hline & 2 & $1.109^{\prime}$ & $0.008^{\prime}$ & $200.4^{\circ}$ & 2 & $0.078^{\prime}$ & $0.008^{\prime}$ & $202.8^{\circ}$ \\
\hline & 3 & $0.430^{\prime}$ & $0.008^{\prime}$ & $79.3^{\circ}$ & 3 & $0.003^{\prime}$ & $0.008^{\prime}$ & $204.3^{\circ}$ \\
\hline & 4 & $0.332^{\prime}$ & $0.005^{\prime}$ & $270.0^{\circ}$ & 4 & $0.004^{\prime}$ & $0.005^{\prime}$ & $255.5^{\circ}$ \\
\hline
\end{tabular}

are at $2 \mathrm{cpd}$ and $1.93227 \mathrm{cpd}$ and are the solar and lunar semi-diurnal waves $\mathbf{S}_{2}$ and $\mathbf{L}_{2}$. The amplitude of the $\mathbf{L}_{2}$ component in the magnetic D-record at Dourbes is about $0.15^{\prime}$. Since there is an annual change in the mean ionospheric conductivity, splitting of the lunar semi-diurnal line is expected. The triplet of peaks at the frequencies $(1.92954,1.93227$, 1.93501) cpd in Fig. 2 is exactly at the frequency of $\mathbf{L}_{2}$ and its annual sidebands and they are accordingly denoted by $\left(\mathbf{L}_{2,-1}, \mathbf{L}_{2}, \mathbf{L}_{2,1}\right)$. This is clear evidence for an annual modulation in the $\mathbf{L}_{2}$ lunar tide. Apparently the splitting of $\mathbf{L}_{2}$ by the semi-annual variation is too weak to rise distinctly above the background noise.

A minor term is also expected in the diurnal band at the frequency of the lunar wave $\mathbf{L}_{1}$. There is an indication in Fig. 1 of a triplet of lines at the frequencies $(0.92954$, $0.93227,0.93501)$ cpd in the vicinity of the wave $\mathbf{L}_{1}$. These peaks are located exactly where the dynamo term $\mathbf{L}_{1}$ and its annual sidebands would be expected. For this reason the lines are denoted by $\left(\mathbf{L}_{1,-1}, \mathbf{L}_{1}, \mathbf{L}_{1,1}\right)$. Together these spectral peaks in Figs. 1 and 2 almost certainly represent splitting of the lunar diurnal and semi-diurnal lines by an annual modulation mechanism. In general, the sidebands of $\mathbf{L}_{n}$ are denoted by $\mathbf{L}_{n, k}, n=1,2, \ldots ; k= \pm 1, \pm 2, \ldots$.

The sidebands of the $1 \mathrm{cpd}$ peak in Fig. 1 at the frequencies $(1+k / 27) \mathrm{cpd}, k=-1,1,2,3$, suggest a mechanism related to solar rotation (Black, 1970). Owing to the recurrence tendency of magnetic storms and disturbances to repeat after a solar rotation there will be a $\sim 27$-day period amplitude modulation of the solar diurnal magnetic variation. The sidebands of $\mathbf{S}_{n}$ at the frequencies $(n+k / 27) \mathrm{cpd}$, $k= \pm 1, \pm 2, \ldots$, due to the modulation by the $\sim 27$-day recurrences and their harmonics, are denoted by $\mathbf{R}_{n, k}$ in Figs. 1 and 2. Such modulation lines are clearly seen in Fig. 1 at frequencies above $1 \mathrm{cpd}$, but the asymmetry with respect to the frequency of $\mathbf{S}_{1}$ is not predicted by a model requiring low-frequency modulation. The width of the 27-day modulation bands of $\mathbf{S}_{1}$, approximately $0.007 \mathrm{cpd}$, contrasts with the width of the solar diurnal peak of $710^{-5} \mathrm{cpd}$, which is the natural width of a sinusoidal signal in the spectrum for the present length of data. This large bandwidth substantiates the view that these sidebands arise from the broad band solar rotation period.

Similar sidebands of the 2 cpd line $\mathbf{S}_{2}$ are also expected. However, no prediction of the relative magnitudes of the two sets of sidebands can be made, so that the apparent absence of the sidebands of $\mathbf{S}_{2}$ at $(2 \pm k / 27) \mathrm{cpd}$ in Fig. 2 does not disprove the solar rotation mechanism. The lunar semi-diurnal magnetic variation, $\mathbf{L}_{2}$, arises from the interaction of the lunar semi-diurnal tide and the mean ionospheric conductivity, and so this lunar line would be expected to show sidebands at $(1.93227 \pm k / 27) \mathrm{cpd}$. However, the 27-day sidebands of the 1 cpd line $\mathbf{S}_{1}$ have an amplitude less than $1 / 10$ that of the $1 \mathrm{cpd}$ line itself, and lines $1 / 10$ of the amplitude of the lunar peak $\mathbf{L}_{2}$ of circa $0.15^{\prime}$ would be completely hidden by the background noise.

\section{Harmonic Analysis of $S_{\mathrm{q}}$ and $L$}

The lunar variations, $\mathbf{L}$, are given in terms of the amplitudes $\ell_{n}$ and phase angles $\lambda_{n}$ of the first four harmonics $\mathbf{L}_{n}$, $n=1,2,3$ and 4, and are represented by Chapman's phase law

$$
L=\sum_{n=1}^{4} \ell_{n} \sin \left(n t-2 v+\lambda_{n}\right)
$$

(Malin and Chapman, 1970), where $v=t-\tau$ is a measure of the phase (or age) of the Moon. Here $t$ denotes mean solar time, reckoned in hours from local midnight, and $\tau$ is mean lunar time, measured in hours from local lower transit of the mean Moon.

The $\mathbf{L}$ variations are masked by the much greater solar 

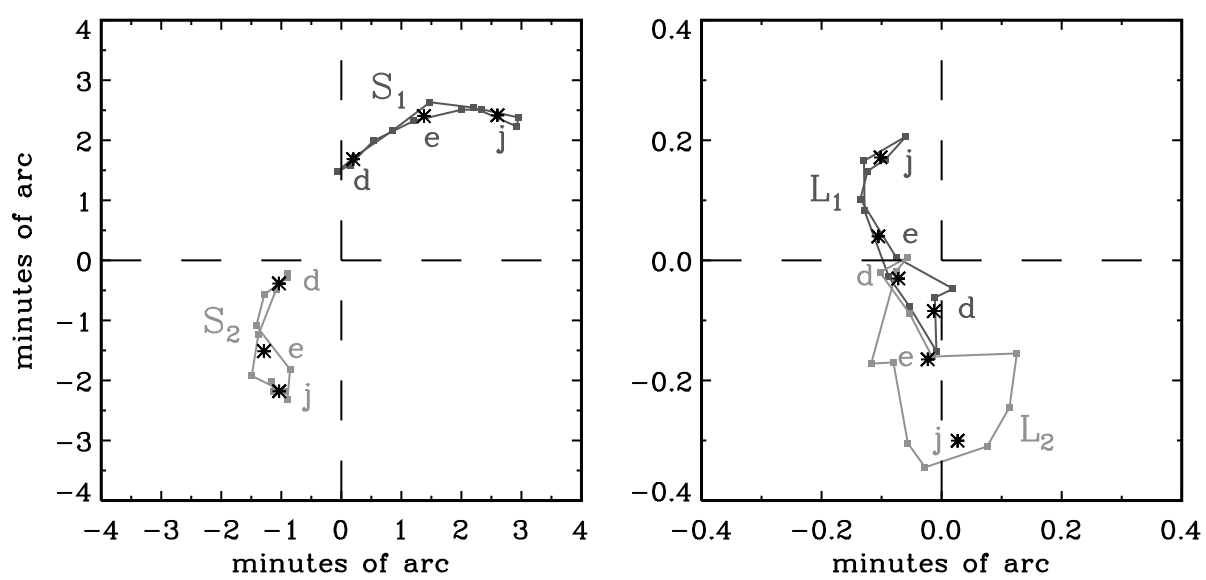

Fig. 3. Harmonic dials showing the month-by-month changes of the diurnal and semi-diurnal harmonics of the solar and lunar daily variations (declination; Chapman-Miller analysis).

daily variations, $\mathbf{S}=\sum_{n} \mathbf{S}_{n}$, which are similarly given in terms of the amplitudes $s_{n}$ and phases $\sigma_{n}$ of the main harmonics $\mathbf{S}_{n}$ as follows:

$$
S=\sum_{n=1}^{4} s_{n} \sin \left(n t+\sigma_{n}\right) .
$$

The frequency of $\mathbf{S}_{n}$ is $n$ cycles per mean solar day and that of $\mathbf{L}_{n}$ is $n-2 / M$, where $M=29.530588$ is the number of solar days in a lunar month. Thus the periods of the first four lunar component variations are as follows: $\mathbf{L}_{1}, 25.74352$ solar hours; $\mathbf{L}_{2}, 12.42060$ solar hours; $\mathbf{L}_{3}, 8.18478$ solar hours; $\mathbf{L}_{4}, 6.10334$ solar hours.

The classical method of analysis is that of Chapman and Miller (1940) as detailed by Malin and Chapman (1970). The main advantage of the Chapman-Miller method is its ability to analyse broken data sequences. To study the seasonal variation of $\mathbf{S}$ and $\mathbf{L}$ it is almost a tradition to separate the data into the Lloyd seasons ( $d$-months: November to February; $e$-months: March, April, September, October; $j$-months: May to August).

The Chapman-Miller method also associates a probable error with the estimates of the amplitude and phase of a particular magnetic variation, which is used to judge the significance of the solar and lunar terms. The vector probable error of the harmonic $\mathbf{S}_{n}$ of $\mathbf{S}$ with amplitude $s_{n}$ and phase $\sigma_{n}$ will be denoted by $r_{n}$. Similarly, the $\mathbf{L}$ data consist of the pairs $\left(\ell_{n}, \lambda_{n}\right)$ and the vector probable errors $\rho_{n}$. The results of a Chapman-Miller analysis are represented in Table 1, giving the amplitudes, vector probable errors and phases of the solar and lunar harmonics, respectively. The first analysis uses all data irrespective of any criterion of selection, whereas the data are subdivided into the Lloyd seasons in the other three analyses. All probable errors are less than $0.02^{\prime}$. Note that the data are analyzed in terms of local time, which means that the phases $\sigma_{n}$ and $\lambda_{n}$ are not corrected for the longitude of the station to compensate for the difference between local time and Greenwich time.

From Table 1, it follows that the solar harmonics $\mathbf{S}_{1}$ and $\mathbf{S}_{2}$ and the lunar harmonics $\mathbf{L}_{1}$ and $\mathbf{L}_{2}$ show an obvious increase in amplitude from $d$-months to $j$-months (winter to summer), the equinoctial amplitude being intermediate between the other two. Since this well-known effect is less conspicuous for the harmonics of higher degree, the further discussion and graphical representations will mainly concern the changes in the amplitudes and phases of $\mathbf{S}_{1}, \mathbf{S}_{2}, \mathbf{L}_{1}$ and $\mathbf{L}_{2}$.

The results of an harmonic analysis may also be represented in a graphical manner. For instance, the pair $\left(s_{n}, \sigma_{n}\right)$ defines a plane vector so that in a plane coordinate system the periodic component $\mathbf{S}_{n}$ is represented by a point having the Cartesian coordinates $\left(s_{n} \cos \sigma_{n}, s_{n} \sin \sigma_{n}\right)$. The vector from the origin to this point has the length $s_{n}$ and its inclination to the horizontal axis is the phase angle $\sigma_{n}$, measured in the counter-clockwise sense. Supposing that a scale of time is inserted on this harmonic dial (or vectogram), measured from the positive horizontal axis in the anti-clockwise direction, the vector which targets the point with polar coordinates $\left(s_{n}, \sigma_{n}\right)$ will indicate the time of the maximum of the sine wave of frequency $n$ cycles per mean solar day.

Suppose that at time $t$, the $n$-th harmonic of the $\mathbf{S}$ and $\mathbf{L}$ variations in the frequency band considered is represented in a plane coordinate system by points with coordinates $\left[s_{n}(t) \cos \sigma_{n}(t), s_{n}(t) \sin \sigma_{n}(t)\right]$ and $\left[\ell_{n}(t) \cos \lambda_{n}(t), \ell_{n}(t) \sin \lambda_{n}(t)\right]$, respectively. A mean solar vector $\left(\bar{s}_{n}, \bar{\sigma}_{n}\right)$ and a mean lunar vector $\left(\bar{\ell}_{n}, \bar{\lambda}_{n}\right)$ can then be computed by taking the average of the individual dial vectors for a given time interval. In particular, the coordinates $\left(\bar{s}_{n} \cos \bar{\sigma}_{n}, \bar{s}_{n} \sin \bar{\sigma}_{n}\right)$ of the mean solar variation vector of the harmonic of degree $n$, defined by the point with polar coordinates $\left(\bar{s}_{n}, \bar{\sigma}_{n}\right)$, are obtained from the arithmetic means of $s_{n}(t) \cos \sigma_{n}(t)$ and $s_{n}(t) \sin \sigma_{n}(t)$.

Next, the 40 years of observations are divided into 12 monthly groups and the mean harmonic constants are obtained for each individual calender month. Figure 3 shows the harmonic dials of the month-by-month variations for the first and second harmonics of $\mathbf{S}$ and $\mathbf{L}$. Mean dial vectors are also computed for the data grouped in the Lloyd seasons; the ' $*$ ' in Fig. 3 indicate the mean dial vectors for the $j-, e$ - and $d$-months. The vectograms in Fig. 3 show that the annual variation in the amplitude of $\mathbf{S}$ presents a maximum in summer with an important phase modulation of the solar daily variation during the year. There also appears to be a significant enhancement of the lunar tide in the $j$-months with a 
Table 2. Declination-Chapman-Miller harmonic analysis. Correlation coefficients $r$ (in \%) and Wolf ratios.

\begin{tabular}{ccccccccc}
\hline $\mathbf{S}$ & $s_{1}$ & $s_{2}$ & $s_{3}$ & $s_{4}$ & $\sigma_{1}$ & $\sigma_{2}$ & $\sigma_{3}$ & $\sigma_{4}$ \\
\hline$r$ & 95.4 & 95.5 & 94.3 & 31.4 & -83.9 & -45.2 & -68.6 & -17.9 \\
$10^{4} M$ & $51 \pm 2$ & $36 \pm 2$ & $54 \pm 2$ & $7 \pm 3$ & $-19 \pm 2$ & $-2 \pm 1$ & $-11 \pm 2$ & $-1 \pm 1$ \\
\hline
\end{tabular}

\begin{tabular}{ccccccccc}
\hline $\mathbf{L}$ & $\ell_{1}$ & $\ell_{2}$ & $\ell_{3}$ & $\ell_{4}$ & $\lambda_{1}$ & $\lambda_{2}$ & $\lambda_{3}$ & $\lambda_{4}$ \\
\hline$r$ & 45.7 & 26.3 & 21.4 & 0.8 & -3.8 & 24.1 & 9.8 & 10.5 \\
$10^{4} M$ & $83 \pm 8$ & $24 \pm 11$ & $17 \pm 11$ & $1 \pm 21$ & $-3 \pm 12$ & $3 \pm 2$ & $5 \pm 8$ & $13 \pm 17$ \\
\hline
\end{tabular}

considerable phase shift of the $\mathbf{L}$ current system between local winter and summer. The total change in phase from winter months to summer months is of the order of $50^{\circ}$ in $\mathbf{S}$ and about $150^{\circ}$ in $\mathbf{L}$. From the point of view of phase modulation, the seasonal variations of $\mathbf{L}$ differ markedly from those of $\mathbf{S}$.

It is convenient to discuss the solar cycle effect on the solar and lunar daily variations in terms of the annual means of the daily dial vectors $\left(s_{n}, \sigma_{n}\right)$ and $\left(\ell_{n}, \lambda_{n}\right)$. The influence of the solar cycle on any of the harmonic terms, $\theta$ (which can be the annual means of either of $s_{n}, \sigma_{n}, \ell_{n}$ or $\lambda_{n}$ ), is expressed numerically by a linear equation of the form

$$
\theta=A+B R \text { or } \theta=A(1+M R), \text { where } M=B / A .
$$

$R$ is the annual mean sunspot number, which is considered here as a representative index of solar activity. The hypothesis of a linear relationship between the harmonic amplitudes and the sunspot number is implicitly based on the physical argument that the main effect of solar activity is to increase the ionospheric conductivity. No significant departures from linearity are found by Chapman et al. (1971) and Malin et al. (1975). It is true that the amplitude of $\mathbf{S}$ increases with sunspot number but there is still some controversy over the response of $\mathbf{L}$ to $R$.

The constants $A$ and $B$ are determined by least squares fitting of a straight line to the annual means of $\theta$ and $R$. Here, $M$ is the Wolf ratio (Chapman et al., 1971; Malin et al., 1975); $10^{4} M$ represents the percentage change in $\theta$ that would accompany a change of $R$ from 0 to 100, a fairly typical sunspot cycle. The probable error of $M$ is deduced using the residuals from the best fitting straight line (Chapman et al., 1971). Values of $10^{4} M$ ( \pm probable error) are given in Table 2. Also included are the correlation coefficients $r$ between the annual means of the amplitudes $\left(s_{n}\right.$ or $\left.\ell_{n}\right)$ and phases $\left(\sigma_{n}\right.$ or $\left.\lambda_{n}\right)$ of $\mathbf{S}_{n}$ and $\mathbf{L}_{n}, n=1,2,3,4$, on the one hand, and the annual means of the sunspot numbers, $R$, on the other hand.

The main harmonic terms show an increase in amplitude with increasing sunspot number for both $\mathbf{S}$ and $\mathbf{L}$. Correlation coefficients and Wolf ratios of the phase angles of $\mathbf{L}$ are small, implying that the sunspot cycle effect on $\mathbf{L}$ is essentially an enhancement of amplitude. As for the solar variation, only the Wolf ratio of the phase angles of $\mathbf{S}_{1}$ and $\mathbf{S}_{3}$ appears to be significantly different from zero. Anyway, the Wolf ratios for $\sigma_{2}$ and $\sigma_{4}$ are below the acceptable significance level. The criterion for judging the significance of the Wolf ratio $M$ is discussed by Chapman et al. (1971).
Although $\mathbf{S}$ and $\mathbf{L}$ are both intensified at sunspot maximum, the results in Table 2 show that the increase of the amplitude of $\mathbf{S}_{1}$ with sunspot number is about double that of the main harmonic $\mathbf{L}_{2}$ of the $\mathbf{L}$ variation. The manifest differences between the solar and lunar Wolf ratios are not readily explained. From a study of 22 stations, the Wolf ratios for ionospheric E-region electron density 'were not only found to be remarkably constant for each station, in the course of the year, they were also found to be uniform the whole world over, the grand average being $0.0033^{\prime}$ (Malin et al., 1975).

The solar cycle influence on $\mathbf{S}$ and $\mathbf{L}$ has been debated in the past. Matsushita (1967) concludes that the current systems responsible for the solar quiet and lunar variations both flow in the E-layer of the ionosphere. Moreover, Matsushita and Maeda (1965) state that $\mathbf{S}$ and $\mathbf{L}$ are similarly influenced during the sunspot cycle. On the other hand, Malin et al. (1975) argue that $\mathbf{S}$ and $\mathbf{L}$ originate at different levels in the ionosphere and propose an F-region location for the external currents associated with $\mathbf{L}$ to account for the apparent differences between the response of $\mathbf{S}$ and $\mathbf{L}$ to $R$.

The lunar tidal movements in the ionosphere are gravitationally induced, so it is reasonable to assume the change of $\mathbf{L}$ with solar cycle to result purely from changes in ionospheric conductivity with $R$. Thus we might expect $10^{4} \mathrm{M}$ to be of the order of 33 for $\ell_{1}$ and $\ell_{2}$. In fact, lunar Wolf ratios (resulting from non-thermal tides) are very different for $\mathbf{L}_{1}$ and $\mathbf{L}_{2}$. Thus it appears that the main parts of $\mathbf{S}$ and $\mathbf{L}$ originate at different levels in the ionosphere (e.g., Green and Malin, 1971; Chapman et al., 1971; Malin et al., 1975).

An adequate representation of the quiet daily variation is usually obtained with only four harmonics of $\mathbf{S}$ and $\mathbf{L}$ since the $24,12,8$ and $6 \mathrm{~h}$ spectral components are shown to rise significantly above the background noise continuum for most observatories (Campbell, 1987). Although the ChapmanMiller method gives a good reconstruction of the $\mathbf{S}_{\mathrm{q}}$ variation at a station when only quiet days are selected, the analysis merely obtains mean harmonic coefficients for a chosen time epoch. When the representative quiet variations are determined for each month of a year, a second Fourier analysis of these monthly coefficients for their annual and semi-annual changes during the year provides a set of cosine and sine coefficients for the quiet year at an observatory (Chapman and Malin, 1970; Campbell, 1987).

The splitting of the solar and lunar lines in Figs. 1 and 2 demonstrates the seasonal modulation of the solar and lunar daily variations. Using continuous data so that the harmonic amplitudes and phases include the influence of all days, a 
spectral analysis of the daily Fourier constants can be performed to investigate the frequency structure of these daily sequences of harmonic parameters (De Meyer, 1986). The Fourier coefficients show a long-term solar cycle contribution as well as a $\sim 27$-day period amplitude variation originating from the solar rotation mechanism. The modulation model to be presented in the next section is a generalization of the traditional Chapman-Miller analysis in the sense that it incorporates directly the continuous time description of the seasonal variation of the harmonic coefficients. Moreover, the effect of changes in solar activity is taken a priori into account by inserting the time sequence of the daily sunspot numbers as a additional input.

\section{Modulation Model}

On close inspection of the spectra in Figs. 1 and 2 it is possible to interpret the extra peaks in the frequency band of the $n$th harmonic of $\mathbf{S}$ and $\mathbf{L}$ in terms of amplitude and phase variations of a sum of two harmonic oscillators with basic carriers at the frequencies of $\mathbf{S}_{n}$ and $\mathbf{L}_{n}$, respectively. The sidebands of $\mathbf{S}_{n}$ are created by the main harmonics of the annual and 27-day modulation periods. Splitting of the lunar peaks $\mathbf{L}_{n}$ by an annual and semi-annual modulation, as well as a sunspot cycle contribution, will be incorporated in the modulation model to be presented.

The model we propose to fit to the band-pass filtered declination data, $D(t)$, is a logical extension of the ChapmanMiller harmonic analysis procedure. Writing $D(t)=S(t)+$ $L(t)$, the combined luni-solar daily variation is a superposition of the solar model, $S(t)$, and the lunar component, $L(t)$, which are represented by

$$
S(t)=\sum_{n=1}^{4} s_{n}(t) \sin \left[\omega_{n}^{S} t+\sigma_{n}(t)\right],
$$

and

$$
L(t)=\sum_{n=1}^{4} \ell_{n}(t) \sin \left[\omega_{n}^{L} t+\lambda_{n}(t)\right],
$$

respectively. Here, $t$ denotes local solar time in hours. The time origin $t=0$ is chosen at the starting point of the observation interval, so that the phases $\sigma_{n}(t)$ and $\lambda_{n}(t)$ relate to January $1,1960,0^{\mathrm{h}}$. The corresponding periods of the fundamental solar and lunar variation lines are $T_{n}^{S}=2 \pi / \omega_{n}^{S}(=$ period $\left.\mathbf{S}_{n}\right)$ and $T_{n}^{L}=2 \pi / \omega_{n}^{L}\left(=\operatorname{period} \mathbf{L}_{n}\right)$. It is emphasized that a model for a variable mean of the data, as considered by De Meyer (1998) for the Wolf sunspot numbers, is not needed in this context since the band-pass filtering in the data processing effectively removes the long-term trend. Nevertheless, the arithmetic mean of the band-pass filtered observations is eliminated.

In general, the amplitude modulation part, $s_{n}(t)$, and phase modulation constituent, $\sigma_{n}(t)$, of the solar variation component are described by an harmonic model of the form

$$
\begin{aligned}
s_{n}(t)= & s_{n, \mathrm{o}}\left[1+M_{n}^{s} R(t)\right] \\
& +\sum_{k=1}^{N_{s_{n}}} c_{n, k}^{s} \sin \left(\omega_{n, k}^{s} t+\varphi_{n, k}^{s}\right), \\
\sigma_{n}(t)= & \sigma_{n, \mathrm{o}}\left[1+M_{n}^{\sigma} R(t)\right]
\end{aligned}
$$

$$
+\sum_{k=1}^{N_{\sigma_{n}}} c_{n, k}^{\sigma} \sin \left(\omega_{n, k}^{\sigma} t+\varphi_{n, k}^{\sigma}\right),
$$

where $R(t)$ denotes the daily sunspot number at time $t$, which is taken to be the same for all hours of the day. For the lunar variations we have an analogous representation:

$$
\begin{aligned}
\ell_{n}(t)= & \ell_{n, \mathrm{o}}\left[1+M_{n}^{\ell} R(t)\right] \\
& +\sum_{k=1}^{N_{\ell_{n}}} c_{n, k}^{\ell} \sin \left(\omega_{n, k}^{\ell} t+\varphi_{n, k}^{\ell}\right), \\
\lambda_{n}(t)= & \lambda_{n, \mathrm{o}}\left[1+M_{n}^{\lambda} R(t)\right] \\
& +\sum_{k=1}^{N_{\lambda_{n}}} c_{n, k}^{\lambda} \sin \left(\omega_{n, k}^{\lambda} t+\varphi_{n, k}^{\lambda}\right)-2 v_{\mathrm{o}} .
\end{aligned}
$$

The associated modulation periods will be denoted by $T_{n, k}^{s}=2 \pi / \omega_{n, k}^{s}, T_{n, k}^{\sigma}=2 \pi / \omega_{n, k}^{\sigma}, T_{n, k}^{\ell}=2 \pi / \omega_{n, k}^{\ell}$, and $T_{n, k}^{\lambda}=2 \pi / \omega_{n, k}^{\lambda}$. Writing $T_{h}=365.242199$ days for the tropic year (Winch and Cunningham, 1972), i.e., the period of variation of the mean longitude $h$ of the Sun, the modulation periods in this context are: $T_{n, k}^{s}=T_{n, k}^{\sigma}=T_{n, k}^{\ell}=T_{n, k}^{\lambda}=T_{h} / k$, for $n=1,2,3,4 ; k=1,2, \ldots$. Note that we have not considered the modulation periods $T_{n, k}^{\ell}$ and $T_{n, k}^{\lambda}$ for $k=3$ and $k=4$, which means that the seasonal change of the $\mathbf{L}$ variation is only described by the first and second harmonic of the annual variation (i.e., $N_{S_{n}}=N_{\sigma_{n}}=4$ and $\left.N_{\ell_{n}}=N_{\lambda_{n}}=2, n=1,2,3,4\right)$.

Using Eq. (3), the solar cycle effect on the solar and lunar daily variations is estimated a posteriori by the Wolf ratios $M$ for the amplitudes and phases obtained by the harmonic analysis. Since we assume a linear dependence on the sunspot numbers $R$, the Wolf ratios $M_{n}^{s}, M_{n}^{\sigma}, M_{n}^{\ell}$, and $M_{n}^{\lambda}$ are now introduced a priori as extra optimization parameters in Eqs. (6), (7), (8), and (9) for the amplitudes and the phases of $\mathbf{S}_{n}$ and $\mathbf{L}_{n}$. To make the results for $s_{n, \mathrm{o}}, \sigma_{n, \mathrm{o}}, \ell_{n, \mathrm{o}}$ and $\lambda_{n, o}$ independent of solar activity the arithmetic mean is eliminated from the sequence of sunspot numbers.

The value of $\lambda_{n}$, defined in Eq. (1), is based on local lunar time and local solar time, whereas the lunar variation model, $L(t)$, in Eq. (5) is completely described in terms of local solar time. This implies that a correction $2 v_{\mathrm{o}}$ is made to the phase $\lambda_{n}(t)$ in Eq. (9), where $v_{\mathrm{o}}$ is the value of the lunar phase $v$ at the initial date of the observing interval (here $\left.2 v_{\mathrm{o}}=62.011^{\circ}\right)$. In this way the resulting values for $\lambda_{n, \mathrm{o}}$ will be directly comparable with the values of the phases $\lambda_{n}$ obtained by the Chapman-Miller harmonic analysis.

In consequence, spectral contributions will appear at the frequencies $\omega_{n}^{S} \pm \omega_{n, k}^{s}$ (amplitude $\frac{1}{2} c_{n, k}^{s}, 1 \leq k \leq N_{s_{n}}$ ), $\omega_{n}^{S} \pm$ $\omega_{n, k}^{\sigma}$ (amplitude $\frac{1}{2} s_{n, o} c_{n, k}^{\sigma}, 1 \leq k \leq N_{\sigma_{n}}$ ), and $\omega_{n}^{S} \pm \omega_{n, k}^{s} \pm \omega_{n, j}^{\sigma}$ (amplitude $\frac{1}{4} c_{n, k}^{s} c_{n, j}^{\sigma}, 1 \leq k \leq N_{s_{n}}, 1 \leq j \leq N_{\sigma_{n}}$ ), arising from the amplitude and phase modulation of the solar line $\mathbf{S}_{n}$ (Coulson, 1955).

Likewise, sidebands of the lunar line $\mathbf{L}_{n}$ will be generated at the frequencies $\omega_{n}^{L} \pm \omega_{n, k}^{\ell}$ (amplitude $\frac{1}{2} c_{n, k}^{\ell}, 1 \leq k \leq$ $N_{\ell_{n}}$ ), $\omega_{n}^{L} \pm \omega_{n, k}^{\lambda}$ (amplitude $\frac{1}{2} \ell_{n, o} c_{n, k}^{\lambda}, 1 \leq k \leq N_{\lambda_{n}}$ ), and $\omega_{n}^{L} \pm \omega_{n, k}^{\ell} \pm \omega_{n, j}^{\lambda}$ (amplitude $\frac{1}{4} c_{n, k}^{\ell} c_{n, j}^{\lambda}, 1 \leq k \leq N_{\ell_{n}}$, (6) $1 \leq j \leq N_{\lambda_{n}}$ ). As a result, sidebands of more than the fourth order would be created, even if $N_{S_{n}}$ and $N_{\sigma_{n}}$ are 4 and $N_{\ell_{n}}$ and $N_{\lambda_{n}}$ are 2. For instance, sidebands of the solar 
Table 3. Declination-Modulation model solar variation.

\begin{tabular}{|c|c|c|c|c|c|c|c|c|}
\hline \multicolumn{9}{|c|}{ Amplitude modulation $s_{n}(t)$ : } \\
\hline$n$ & $s_{n, \mathrm{o}}$ & $\Delta s_{n, 0}$ & $k$ & $T_{n, k}^{s}$ & $c_{n, k}^{s}$ & $\Delta c_{n, k}^{s}$ & $\varphi_{n, k}^{s}$ & $\Delta \varphi_{n, k}^{s}$ \\
\hline \multirow[t]{4}{*}{1} & $2.7034^{\prime}$ & $0.0038^{\prime}$ & 1 & $T_{h}^{\dagger}$ & $1.1139^{\prime}$ & $0.0040^{\prime}$ & $278.321^{\circ}$ & $0.289^{\circ}$ \\
\hline & & & 2 & $T_{h} / 2$ & $0.1079^{\prime}$ & $0.0006^{\prime}$ & $295.767^{\circ}$ & $1.315^{\circ}$ \\
\hline & & & 3 & $T_{h} / 3$ & $0.0216^{\prime}$ & $0.0001^{\prime}$ & $7.073^{\circ}$ & $0.044^{\circ}$ \\
\hline & & & 4 & $T_{h} / 4$ & $0.0370^{\prime}$ & $0.0002^{\prime}$ & $146.526^{\circ}$ & $0.901^{\circ}$ \\
\hline \multirow[t]{4}{*}{2} & $1.8389^{\prime}$ & $0.0038^{\prime}$ & 1 & $T_{h}$ & $0.7747^{\prime}$ & $0.0037^{\prime}$ & $275.101^{\circ}$ & $0.406^{\circ}$ \\
\hline & & & 2 & $T_{h} / 2$ & $0.2392^{\prime}$ & $0.0013^{\prime}$ & $256.340^{\circ}$ & $0.939^{\circ}$ \\
\hline & & & 3 & $T_{h} / 3$ & $0.0928^{\prime}$ & $0.0005^{\prime}$ & $172.137^{\circ}$ & $1.023^{\circ}$ \\
\hline & & & 4 & $T_{h} / 4$ & $0.0220^{\prime}$ & $0.0001^{\prime}$ & $30.275^{\circ}$ & $0.189^{\circ}$ \\
\hline \multirow[t]{4}{*}{3} & $0.8000^{\prime}$ & $0.0033^{\prime}$ & 1 & $T_{h}$ & $0.2457^{\prime}$ & $0.0019^{\prime}$ & $273.118^{\circ}$ & $1.082^{\circ}$ \\
\hline & & & 2 & $T_{h} / 2$ & $0.3284^{\prime}$ & $0.0021^{\prime}$ & $273.820^{\circ}$ & $0.869^{\circ}$ \\
\hline & & & 3 & $T_{h} / 3$ & $0.0578^{\prime}$ & $0.0005^{\prime}$ & $181.752^{\circ}$ & $1.333^{\circ}$ \\
\hline & & & 4 & $T_{h} / 4$ & $0.0573^{\prime}$ & $0.0004^{\prime}$ & $70.549^{\circ}$ & $0.477^{\circ}$ \\
\hline \multirow[t]{4}{*}{4} & $0.2626^{\prime}$ & $0.0020^{\prime}$ & 1 & $T_{h}$ & $0.1701^{\prime}$ & $0.0011^{\prime}$ & $101.714^{\circ}$ & $0.705^{\circ}$ \\
\hline & & & 2 & $T_{h} / 2$ & $0.1709^{\prime}$ & $0.0012^{\prime}$ & $266.701^{\circ}$ & $1.326^{\circ}$ \\
\hline & & & 3 & $T_{h} / 3$ & $0.0612^{\prime}$ & $0.0004^{\prime}$ & $286.019^{\circ}$ & $1.756^{\circ}$ \\
\hline & & & 4 & $T_{h} / 4$ & $0.0515^{\prime}$ & $0.0004^{\prime}$ & $68.156^{\circ}$ & $0.469^{\circ}$ \\
\hline
\end{tabular}

\begin{tabular}{|c|c|c|c|c|c|c|c|c|}
\hline \multicolumn{9}{|c|}{ Phase modulation $\sigma_{n}(t)$ : } \\
\hline$n$ & $\sigma_{n, \mathrm{o}}$ & $\Delta \sigma_{n, \mathrm{o}}$ & $k$ & $T_{n, k}^{\sigma}$ & $c_{n, k}^{\sigma}$ & $\Delta c_{n, k}^{\sigma}$ & $\varphi_{n, k}^{\sigma}$ & $\Delta \varphi_{n, k}^{\sigma}$ \\
\hline \multirow[t]{4}{*}{1} & $62.325^{\circ}$ & $0.063^{\circ}$ & 1 & $T_{h}$ & $23.320^{\circ}$ & $0.073^{\circ}$ & $100.474^{\circ}$ & $0.203^{\circ}$ \\
\hline & & & 2 & $T_{h} / 2$ & $1.551^{\circ}$ & $0.009^{\circ}$ & $125.822^{\circ}$ & $0.731^{\circ}$ \\
\hline & & & 3 & $T_{h} / 3$ & $2.238^{\circ}$ & $0.012^{\circ}$ & $88.327^{\circ}$ & $0.462^{\circ}$ \\
\hline & & & 4 & $T_{h} / 4$ & $2.035^{\circ}$ & $0.011^{\circ}$ & $208.091^{\circ}$ & $0.976^{\circ}$ \\
\hline \multirow[t]{4}{*}{2} & $223.878^{\circ}$ & $0.106^{\circ}$ & 1 & $T_{h}$ & $27.372^{\circ}$ & $0.111^{\circ}$ & $267.266^{\circ}$ & $0.319^{\circ}$ \\
\hline & & & 2 & $T_{h} / 2$ & $6.424^{\circ}$ & $0.035^{\circ}$ & $279.145^{\circ}$ & $1.016^{\circ}$ \\
\hline & & & 3 & $T_{h} / 3$ & $3.733^{\circ}$ & $0.020^{\circ}$ & $85.897^{\circ}$ & $0.482^{\circ}$ \\
\hline & & & 4 & $T_{h} / 4$ & $3.891^{\circ}$ & $0.024^{\circ}$ & $215.424^{\circ}$ & $0.981^{\circ}$ \\
\hline \multirow[t]{4}{*}{3} & $78.558^{\circ}$ & $0.164^{\circ}$ & 1 & $T_{h}$ & $7.049^{\circ}$ & $0.056^{\circ}$ & $239.873^{\circ}$ & $1.094^{\circ}$ \\
\hline & & & 2 & $T_{h} / 2$ & $4.370^{\circ}$ & $0.028^{\circ}$ & $106.308^{\circ}$ & $0.784^{\circ}$ \\
\hline & & & 3 & $T_{h} / 3$ & $5.642^{\circ}$ & $0.043^{\circ}$ & $79.486^{\circ}$ & $0.580^{\circ}$ \\
\hline & & & 4 & $T_{h} / 4$ & $5.385^{\circ}$ & $0.032^{\circ}$ & $254.758^{\circ}$ & $1.278^{\circ}$ \\
\hline \multirow[t]{4}{*}{4} & $260.616^{\circ}$ & $0.606^{\circ}$ & 1 & $T_{h}$ & $26.698^{\circ}$ & $0.153^{\circ}$ & $129.174^{\circ}$ & $0.805^{\circ}$ \\
\hline & & & 2 & $T_{h} / 2$ & $29.991^{\circ}$ & $0.192^{\circ}$ & $289.855^{\circ}$ & $1.323^{\circ}$ \\
\hline & & & 3 & $T_{h} / 3$ & $22.563^{\circ}$ & $0.181^{\circ}$ & $123.798^{\circ}$ & $0.766^{\circ}$ \\
\hline & & & 4 & $T_{h} / 4$ & $12.699^{\circ}$ & $0.101^{\circ}$ & $317.630^{\circ}$ & $1.800^{\circ}$ \\
\hline
\end{tabular}

${ }^{\dagger} T_{h}=365.242199$ days.

semi-diurnal line, $\mathbf{S}_{2}$, up to the sixth order are noticed in the amplitude spectrum in Fig. 2. This could explain the different observed amplitudes of the symmetrical sidebands, respectively surrounding the lines $\mathbf{S}_{1}, \mathbf{S}_{2}, \mathbf{L}_{1}$ and $\mathbf{L}_{2}$ in Figs. 1 and 2 , because different amplitude and phase modulation periods may contribute power to the same frequency.

The fundamental concept of the modulation model therefore consists of the sum of harmonic oscillators with basic carriers having the primary frequencies $\left(\omega_{n}^{S}\right.$ and $\omega_{n}^{L}$, $n=1,2,3,4)$ of the solar and lunar daily variations, respectively, which are being amplitude and phase modulated by fluctuations that can be described by a sum of trigonometric terms with frequencies fixed by the annual variation and its harmonics. Representing the solar cycle contribution by the daily sunspot numbers in a way which is compatible with Eq. (3), Wolf ratios for the amplitudes and the phases of the solar and lunar variations are introduced as additional regression coefficients to be optimized together with the other model parameters. This means that the effect of changes in solar activity on the harmonic terms is incorporated by using the sunspot numbers as a supplemental input into the modulation model. 
Table 4. Declination-Modulation model lunar variation.

\begin{tabular}{|c|c|c|c|c|c|c|c|c|}
\hline \multicolumn{9}{|c|}{ Amplitude modulation $\ell_{n}(t)$ : } \\
\hline$n$ & $\ell_{n, \mathrm{o}}$ & $\Delta \ell_{n, \mathrm{o}}$ & $k$ & $T_{n, k}^{\ell}$ & $c_{n, k}^{\ell}$ & $\Delta c_{n, k}^{\ell}$ & $\varphi_{n, k}^{\ell}$ & $\Delta \varphi_{n, k}^{\ell}$ \\
\hline \multirow[t]{2}{*}{1} & $0.1096^{\prime}$ & $0.0007^{\prime}$ & 1 & $T_{h}^{\dagger}$ & $0.0690^{\prime}$ & $0.0006^{\prime}$ & $286.413^{\circ}$ & $2.306^{\circ}$ \\
\hline & & & 2 & $T_{h} / 2$ & $0.0227^{\prime}$ & $0.0002^{\prime}$ & $79.695^{\circ}$ & $0.756^{\circ}$ \\
\hline \multirow[t]{2}{*}{2} & $0.1854^{\prime}$ & $0.0013^{\prime}$ & 1 & $T_{h}$ & $0.1302^{\prime}$ & $0.0009^{\prime}$ & $273.695^{\circ}$ & $1.634^{\circ}$ \\
\hline & & & 2 & $T_{h} / 2$ & $0.0163^{\prime}$ & $0.0002^{\prime}$ & $162.470^{\circ}$ & $1.310^{\circ}$ \\
\hline \multirow[t]{2}{*}{3} & $0.0659^{\prime}$ & $0.0006^{\prime}$ & 1 & $T_{h}$ & $0.0638^{\prime}$ & $0.0005^{\prime}$ & $276.267^{\circ}$ & $2.105^{\circ}$ \\
\hline & & & 2 & $T_{h} / 2$ & $0.0150^{\prime}$ & $0.0001^{\prime}$ & $284.212^{\circ}$ & $2.463^{\circ}$ \\
\hline \multirow[t]{2}{*}{4} & $0.0213^{\prime}$ & $0.0002^{\prime}$ & 1 & $T_{h}$ & $0.0041^{\prime}$ & $0.0001^{\prime}$ & $289.311^{\circ}$ & $2.232^{\circ}$ \\
\hline & & & 2 & $T_{h} / 2$ & $0.0134^{\prime}$ & $0.0001^{\prime}$ & $304.897^{\circ}$ & $2.131^{\circ}$ \\
\hline
\end{tabular}

\begin{tabular}{|c|c|c|c|c|c|c|c|c|}
\hline \multicolumn{9}{|c|}{ Phase modulation $\lambda_{n}(t)$ : } \\
\hline$n$ & $\lambda_{n, \mathrm{o}}$ & $\Delta \lambda_{n, \mathrm{o}}$ & $k$ & $T_{n, k}^{\lambda}$ & $c_{n, k}^{\lambda}$ & $\Delta c_{n, k}^{\lambda}$ & $\varphi_{n, k}^{\lambda}$ & $\Delta \varphi_{n, k}^{\lambda}$ \\
\hline \multirow[t]{2}{*}{1} & $195.011^{\circ}$ & $1.049^{\circ}$ & 1 & $T_{h}$ & $82.577^{\circ}$ & $0.593^{\circ}$ & $96.741^{\circ}$ & $0.693^{\circ}$ \\
\hline & & & 2 & $T_{h} / 2$ & $12.690^{\circ}$ & $0.122^{\circ}$ & $134.639^{\circ}$ & $1.103^{\circ}$ \\
\hline \multirow[t]{2}{*}{2} & $262.291^{\circ}$ & $1.007^{\circ}$ & 1 & $T_{h}$ & $48.200^{\circ}$ & $0.393^{\circ}$ & $239.389^{\circ}$ & $1.425^{\circ}$ \\
\hline & & & 2 & $T_{h} / 2$ & $19.042^{\circ}$ & $0.161^{\circ}$ & $275.637^{\circ}$ & $2.273^{\circ}$ \\
\hline \multirow[t]{2}{*}{3} & $174.581^{\circ}$ & $1.146^{\circ}$ & 1 & $T_{h}$ & $78.184^{\circ}$ & $0.657^{\circ}$ & $120.435^{\circ}$ & $0.975^{\circ}$ \\
\hline & & & 2 & $T_{h} / 2$ & $43.699^{\circ}$ & $0.419^{\circ}$ & $124.792^{\circ}$ & $1.214^{\circ}$ \\
\hline \multirow[t]{2}{*}{4} & $300.535^{\circ}$ & $2.281^{\circ}$ & 1 & $T_{h}$ & $65.486^{\circ}$ & $0.589^{\circ}$ & $130.876^{\circ}$ & $1.126^{\circ}$ \\
\hline & & & 2 & $T_{h} / 2$ & $69.799^{\circ}$ & $0.530^{\circ}$ & $241.318^{\circ}$ & $2.006^{\circ}$ \\
\hline
\end{tabular}

${ }^{\dagger} T_{h}=365.242199$ days.

Table 5. Declination-Modulation model. Wolf ratios.

\begin{tabular}{ccrcc}
\hline$n$ & $10^{4} M_{n}^{s}$ & \multicolumn{1}{c}{$10^{4} M_{n}^{\sigma}$} & $10^{4} M_{n}^{\ell}$ & $10^{4} M_{n}^{\lambda}$ \\
\hline 1 & $26.93 \pm 0.19$ & $-14.53 \pm 0.09$ & $31.57 \pm 0.27$ & $-0.94 \pm 0.01$ \\
2 & $26.93 \pm 0.21$ & $-0.92 \pm 0.01$ & $31.24 \pm 0.30$ & $-1.90 \pm 0.02$ \\
3 & $32.83 \pm 0.26$ & $-2.25 \pm 0.02$ & $34.02 \pm 0.30$ & $-1.99 \pm 0.02$ \\
4 & $24.99 \pm 0.25$ & $-2.28 \pm 0.02$ & $35.83 \pm 0.23$ & $-1.11 \pm 0.01$ \\
\hline
\end{tabular}

Initial estimates of the amplitudes $\left(s_{n, 0}, c_{n, k}^{s}, c_{n, k}^{\sigma} ; \ell_{n, 0}\right.$, $\left.c_{n, k}^{\ell}, c_{n, k}^{\lambda}\right)$ and phases $\left(\sigma_{n, 0}, \varphi_{n, k}^{s}, \varphi_{n, k}^{\sigma} ; \lambda_{n, 0}, \varphi_{n, k}^{\ell}, \varphi_{n, k}^{\lambda}\right)$ are obtained as follows. Figure 3 visualizes the results of an harmonic Chapman-Miller analysis for each calender month. For instance, since the frequencies $\omega_{n, k}^{s}$ are chosen a priori estimates of the amplitudes, $s_{n, \mathrm{o}}$ and $c_{n, k}^{s}$, and phases, $\varphi_{n, k}^{s}$, of the model (6) for the amplitude factor $s_{n}(t)$ result from least squares fitting of a trigonometric model of the form

$$
s_{n, 0}+\sum_{k=1}^{4}\left(a_{n, k}^{s} \cos \omega_{n, k}^{s} t+b_{n, k}^{s} \sin \omega_{n, k}^{s} t\right)
$$

to the 12 monthly values of the amplitude of $\mathbf{S}_{n}$ visualized in Fig. 3. The amplitudes $c_{n, k}^{s}$ and phase $\varphi_{n, k}^{s}$ are readily obtained from the estimated values of $a_{n, k}^{s}$ and $b_{n, k}^{s}$. The value 0.0033 is taken as the initial estimate of the Wolf $M$ ratios (Malin et al., 1975).

These starting approximations are used for a non-linear optimization of the model parameters, which are estimated by minimizing the sum of squares of the differences between the filtered declination data and the sum of the synthetic values $S(t)$ and $L(t)$ obtained from Eqs. (4) and (5). For a detailed description of the theoretical basis of the maximum neighbourhood technique the reader is referred to Marquardt (1963). Moreover, as the computer program obtains and improves an approximation to the first derivative matrix following the ideas of Broyden (1967), Marquardt's method also provides standard errors of the parameter estimates.

\section{Results of the Modulation Model}

The optimized parameters of the modulation model for the declination data are summarized in Table 3 and Table 4, respectively for the solar and lunar variations, and the Wolf ratios ( \pm probable error) are compiled in Table 5 . The number of digits is large relative to the expected confidence intervals of the physical parameters and only relate to the computer output. The columns $\Delta$ refer to the standard errors of the estimated parameters by the non-linear optimization method. Note that the phases are not corrected for the longitude of the 


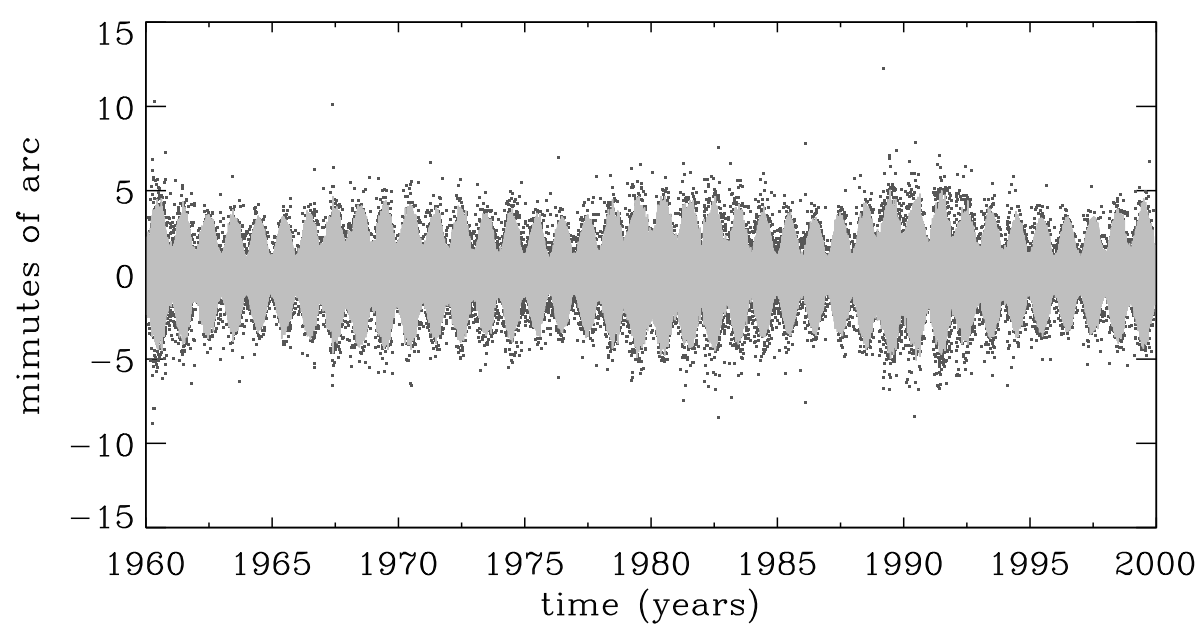

Fig. 4. Declination data and model values (modulation model: data, model).

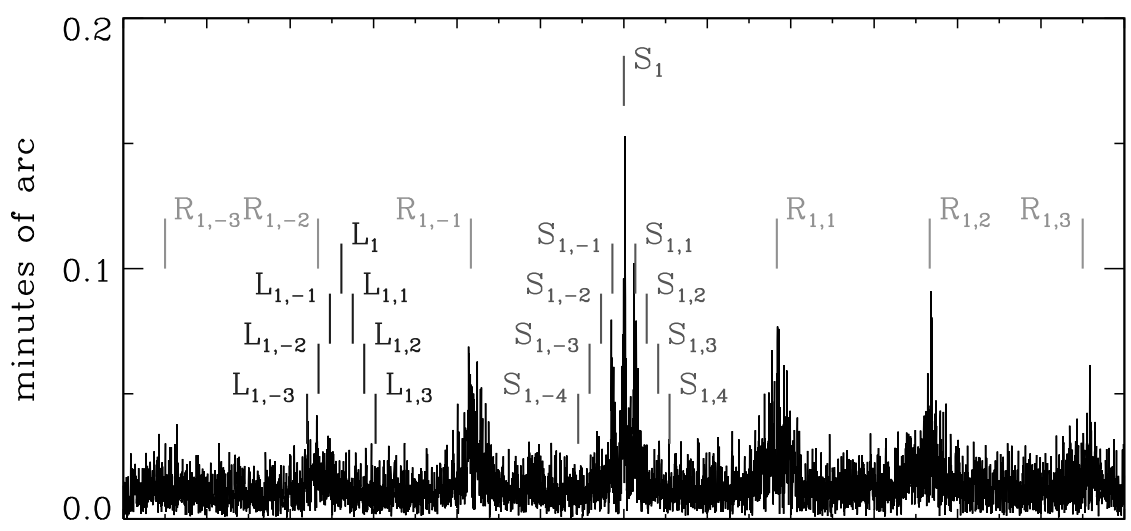

0.880 .900 .920 .940 .960 .981 .001 .021 .041 .061 .081 .101 .12

frequency (cpd)

Fig. 5. Residual amplitude spectrum of the declination in the diurnal band.

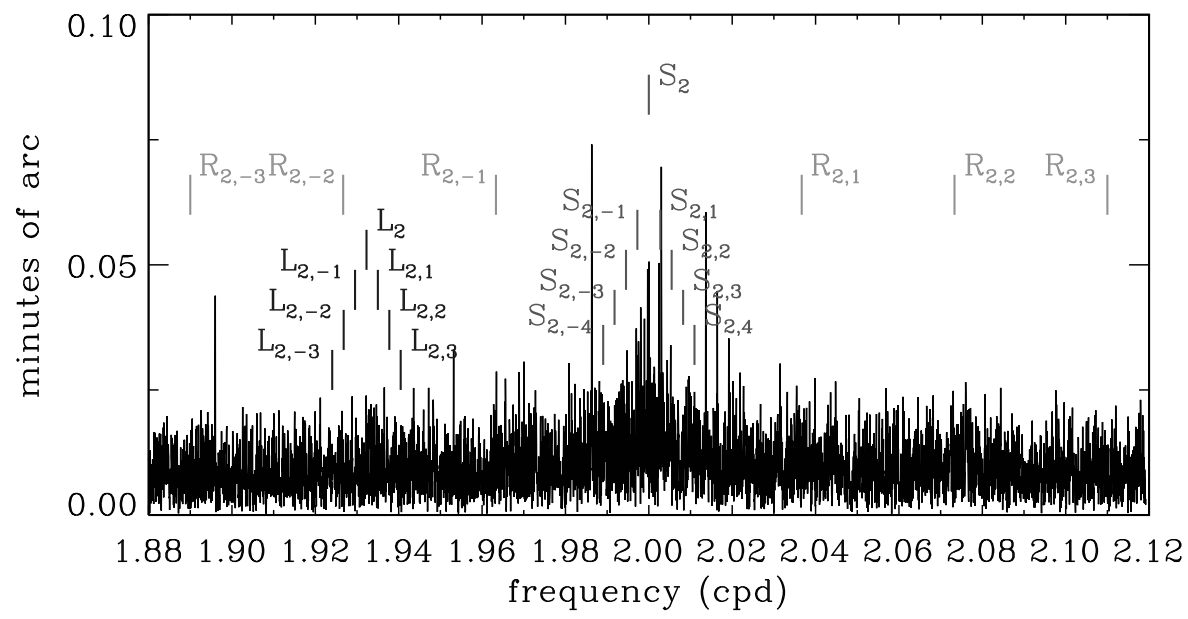

Fig. 6. Residual amplitude spectrum of the declination in the semi-diurnal band.

Dourbes observatory so that phases refer to local solar time. The model accounts for $82.7 \%$ of the total sum of squares of the filtered observations and the residual standard deviation is estimated at $1.16^{\prime}$. The band-pass filtered data and model values are visualized in Fig. 4, whereas Figures 5 and 6 show the residual periodograms, converted to amplitude on the ordinate scale, around 1 and $2 \mathrm{cpd}$, respectively. These diagrams are to be compared with Figs. 1 and 2. Whereas the residual spectra in Figs. 5 and 6 contain some small peaks, the signal-to-noise ratio in the peaks is of the order of 10 and it is concluded that the filtered declination data are essentially reduced to white noise by the algorithm. 

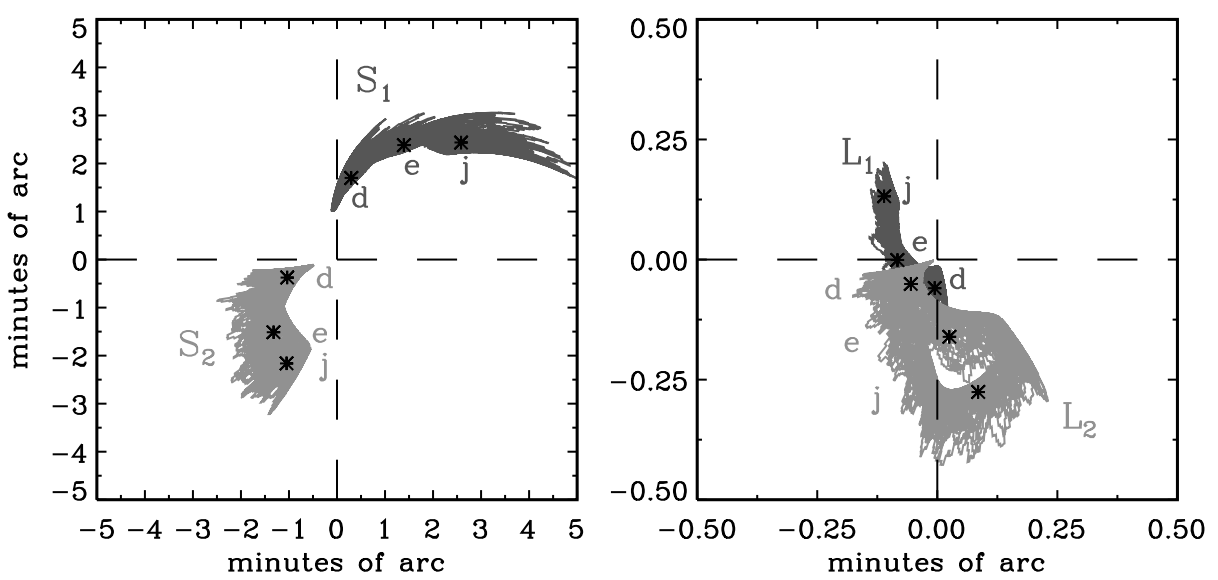

Fig. 7. Vectograms showing the time changes of the diurnal and semi-diurnal components of the solar and lunar daily variations (declination; modulation model.)

Table 6. Declination-Modulation model. Ratios of seasonal amplitude to mean amplitude. Differences of seasonal phase and mean phase.

\begin{tabular}{cccccrrrrr}
\hline $\mathbf{S}$ & $s_{1}$ & $s_{2}$ & $s_{3}$ & \multicolumn{1}{c}{$s_{4}$} & $\sigma_{1}$ & \multicolumn{1}{c}{$\sigma_{2}$} & \multicolumn{1}{c}{$\sigma_{3}$} & \multicolumn{1}{c}{$\sigma_{4}$} \\
\hline$j$-months & 1.36 & 1.36 & 1.08 & 0.14 & $-13^{\mathrm{o}}$ & $14^{\mathrm{o}}$ & $8^{\mathrm{o}}$ & $-12^{\circ}$ \\
$e$-months & 1.06 & 1.14 & 1.38 & 1.65 & $3^{\mathrm{o}}$ & $-1^{\mathrm{o}}$ & $-6^{\mathrm{o}}$ & $5^{\mathrm{o}}$ \\
$d$-months & 0.66 & 0.62 & 0.55 & 1.24 & $23^{\mathrm{o}}$ & $-30^{\mathrm{o}}$ & $-3^{\mathrm{o}}$ & $-6^{\mathrm{o}}$ \\
\hline
\end{tabular}

\begin{tabular}{ccccccrrr}
\hline $\mathbf{L}$ & $\ell_{1}$ & $\ell_{2}$ & $\ell_{3}$ & $\ell_{4}$ & \multicolumn{1}{c}{$\lambda_{1}$} & \multicolumn{1}{c}{$\lambda_{2}$} & \multicolumn{1}{c}{$\lambda_{3}$} & $\lambda_{4}$ \\
\hline$j$-months & 2.42 & 1.76 & 1.85 & 1.43 & $-30^{\circ}$ & $11^{\circ}$ & $-1^{\circ}$ & $-57^{\circ}$ \\
$e$-months & 1.17 & 0.99 & 1.01 & 1.97 & $21^{\circ}$ & $2^{\circ}$ & $-1^{\circ}$ & $44^{\circ}$ \\
$d$-months & 0.84 & 0.46 & 0.14 & 0.81 & $105^{\circ}$ & $-57^{\circ}$ & $29^{\circ}$ & $-11^{\circ}$ \\
\hline
\end{tabular}

\section{Discussion}

The models $S(t)$ and $L(t)$ in Eqs. (4) and (5) can be thought of in geometrical terms, as, for example, with the aid of vector diagrams. For instance, the solar term $S(t)$ is specified by the time variation of the amplitudes $s_{n}(t)$ and the associated phase angles $\sigma_{n}(t)$. At time $t$, the $n$-th harmonic of the $\mathbf{S}$ and $\mathbf{L}$ variations is represented in a plane coordinate system by points with coordinates $\left[s_{n}(t) \cos \sigma_{n}(t), s_{n}(t) \sin \sigma_{n}(t)\right]$ and $\left[\ell_{n}(t) \cos \lambda_{n}(t), \ell_{n}(t) \sin \lambda_{n}(t)\right]$, respectively. To proceed with a coherent notation the constituents of the solar and lunar variation models $S(t)$ and $L(t)$ will now be denoted in the frequency band $n$ as $\mathbf{S}_{n}$ and $\mathbf{L}_{n}$, respectively. The symbols $\left[s_{n}(t), \sigma_{n}(t)\right]$ used in the modulation model (4) will be interpreted as the variation in time of the harmonic coefficients $\left(s_{n}, \sigma_{n}\right)$ for $\mathbf{S}_{n}$. In like manner, $\left[\ell_{n}(t), \lambda_{n}(t)\right]$ are related to $\left(\ell_{n}, \lambda_{n}\right)$ for $\mathbf{L}_{n}$. A mean solar dial vector $\left(\bar{s}_{n}, \bar{\sigma}_{n}\right)$ and a mean lunar vector $\left(\bar{\ell}_{n}, \bar{\lambda}_{n}\right)$ can then be computed by taking the average of the dial vectors for a given time interval. The day-to-day variability of the dial vectors $\left[s_{n}(t), \sigma_{n}(t)\right]$ and $\left[\ell_{n}(t), \lambda_{n}(t)\right]$ is shown in the vectograms of Fig. 7 .

We computed the mean dial vectors for the three Lloyd seasons $j, e, d$, which are also shown in Fig. 7; moreover, the mean amplitude and phase for the $\mathbf{S}$ and $\mathbf{L}$ variations were obtained using all data. As a measure of the seasonal variation in a form suitable for comparison between $\mathbf{S}$ and $\mathbf{L}$, we adopt the ratio of the seasonal amplitude to the overall amplitude; the difference between the seasonal phase and the mean phase is also examined. The seasonal amplitude ratios and phase differences of $\mathbf{S}$ and $\mathbf{L}$ are given in Table 6 .

For $\mathbf{S}_{1}$ and $\mathbf{S}_{2}$, the amplitudes behave as expected, being greatest for the summer months and least for the winter months. The mean ratio of summer to winter amplitude (obtained from the summer/mean and winter/mean ratios) are 2.06 and 2.18 for $\mathbf{S}_{1}$ and $\mathbf{S}_{2}$, respectively. It is interesting to see that the $e$-month amplitude only slightly exceeds the overall mean amplitude. As was found for $\mathbf{S}$, the lunar harmonics $\mathbf{L}_{1}$ and $\mathbf{L}_{2}$ for the magnetic declination also show a marked increase in amplitude from $d$-months to $j$-months, the equinoctial ratio being again intermediate between the other two. The amplitude ratio of $\mathbf{L}_{2}$ in Table 6 is always smaller than the corresponding ratio of $\mathbf{L}_{1}$. The percentage change in amplitude from summer to winter is about the same for $\mathbf{S}_{1}$ and $\mathbf{S}_{2}$, but it is greater for the $\mathbf{L}$ variation, the summer to winter ratio being 2.89 and 3.85 for $\mathbf{L}_{1}$ and $\mathbf{L}_{2}$, respectively. Comparing these values with the corresponding ratios for $\mathbf{S}_{1}$ and $\mathbf{S}_{2}$ we conclude that the amplitude ratios for $\mathbf{L}$ are some 50 per cent greater than the solar ratios. This result confirms that the seasonal change of $\mathbf{L}$ noticeably exceeds that of $\mathbf{S}$.

Figure 7 displays the explicit increase in amplitude of the solar and lunar variations from winter to summer. The phase angles of $\mathbf{S}_{1}$ and $\mathbf{L}_{1}$ vary in the clockwise sense from $d$ months to $j$-months (maximum earlier in summer, later in winter), whereas the phases of $\mathbf{S}_{2}$ and $\mathbf{L}_{2}$ vary in the opposite sense. Also, the exceptional change in phase of $\mathbf{L}_{2}$ from $d$ - 

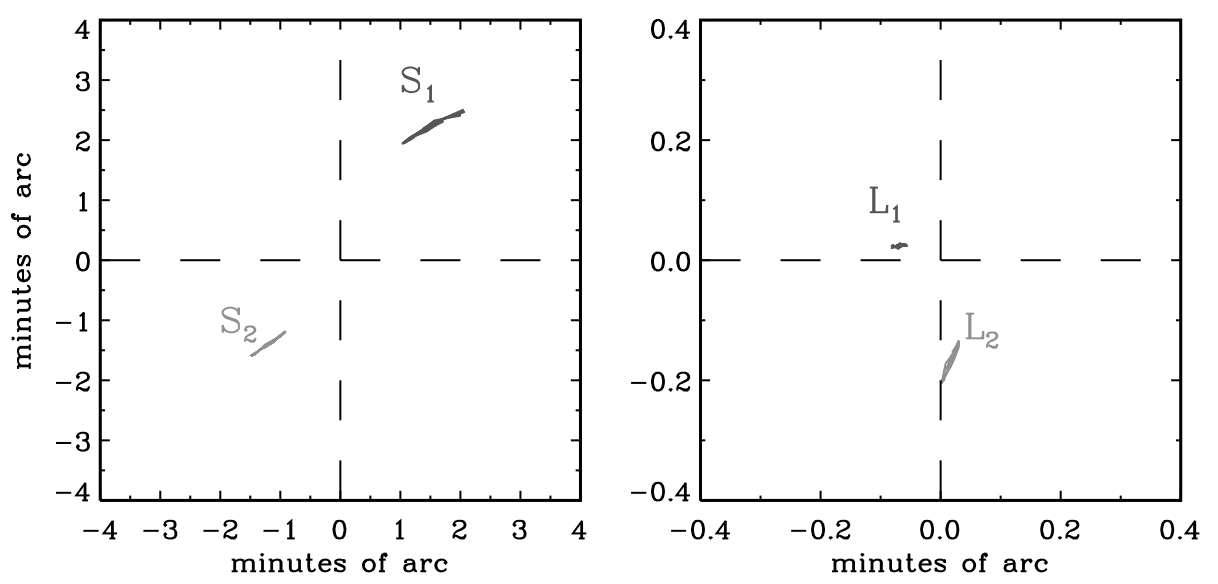

Fig. 8. Harmonic dials of year-by-year changes of the diurnal and semi-diurnal components of the solar and lunar daily variations (declination; modulation model).

months to $j$-months is obvious. The total change in phase from winter months to summer months is of the order of $50^{\circ}$ in $\mathbf{S}$ and about $140^{\circ}$ in $\mathbf{~ L}$. Consequently, there exists an important phase change in the $\mathbf{L}$ current system between local winter and summer and it is about three times that of the $\mathbf{S}$ system.

The effect of solar activity on the amplitude and phase of the solar and lunar variations, obtained from a ChapmanMiller harmonic analysis, is estimated a posteriori by the Wolf ratio $M$ in Eq. (3). Postulating a linear relationship between the annual means of the harmonic coefficients and the annual sunspot numbers the Wolf ratio is defined as the ratio of the least squares estimates of the slope $B$ and the intercept $A$. In this way the results in Table 2 were obtained. However, $\mathbf{S}$ and $\mathbf{L}$ present an important seasonal variation and also depend significantly on the sunspot number, whereas the linear equation is fitted to data where both effects intervene. As can be seen from Table 6 , the amplitudes and phases of the $\mathbf{S}$ and $\mathbf{L}$ variations exhibit a strong seasonal dependence. Even if the slope $B$ is found to be the same for the three Lloyd's seasons, the intercept $A$ would exhibit a strong seasonal dependence and the Wolf ratio $M$ would be concluded to be largely dependent on the season.

On the other hand, Wolf ratios are incorporated a priori in the time description of the amplitude and phase terms in the modulation model through additional regression coefficients and are optimized independently by minimizing the residual sum of squares. In this way the Wolf ratios in Eqs. (6), (7), (8), and (9) are only indirectly related to the intercepts $s_{n, \mathrm{o}}$, $\sigma_{n, \mathrm{o}}, \ell_{n, \mathrm{o}}$, and $\lambda_{n, \mathrm{o}}$, respectively. In consequence, the numerical outcomes of the estimation of the sunspot cycle effect on the solar and lunar daily variations, respectively obtained by the harmonic analysis and the modulation model, may turn out to be different.

The Wolf ratios estimated by the modulation model are summarized in Table 5. The standard errors of the $M$ values are unexpectedly small. However, computational problems frequently arise in non-linear minimization techniques when calculating adequate error estimates for the optimized model parameters. After the observational equations have been linearized by retaining only the terms through the first derivative in a Taylor expansion, changes of the model parame- ters are determined using a least squares procedure. These changes are then added to the previous parameters, and the iteration scheme is continued until the desired convergence of the residual sum of squares is reached. Denoting by $\boldsymbol{F}$ the estimated first derivative matrix of the original set of non-linear observational equations with respect to the model parameters (Broyden, 1967) and by $\boldsymbol{F}^{\prime}$ the transpose of $\boldsymbol{F}$, the inverse $\left(\boldsymbol{F}^{\prime} \boldsymbol{F}\right)^{-1}$ of the cross-product matrix $\boldsymbol{F}^{\prime} \boldsymbol{F}$ is used to estimate the linearized covariance matrix for the parameters. It is often found that the normal matrix $\boldsymbol{F}^{\prime} \boldsymbol{F}$ is ill-conditioned near the minimum of the objective function in parameter space (Tarantola and Valette, 1982), leading to poorly estimated standard errors for the model parameters. Therefore, truncation of the numerical outcomes to significant digits was avoided.

From Table 5 it follows that the values of $10^{4} \mathrm{M}$ for the amplitude of the $\mathbf{L}$ variations are only slightly greater than the corresponding Wolf ratios for the $\mathbf{S}$ variations, ratifying the view of Matsushita and Maeda (1965) that $\mathbf{S}$ and $\mathbf{L}$ are similarly influenced during the solar cycle. However, non-linear optimization problems may be very sensitive to the choice of the starting estimates of the model parameters. With a view to check the initial value effect, the non-linear iteration procedure was restarted by varying the initial approximation to the Wolf ratios $M$ in the neighbourhood of 0.0033 , which is the mean value for ionospheric E-region electron density (Malin et al., 1975). Yet, it was found that starting values differing up to 10 per cent of 0.0033 resulted in nearly the same Wolf ratios as compiled in Table 5. The Wolf ratios of the phase angles $\sigma_{n}$ and $\lambda_{n}$ are small, implying that the solar cycle effect on $\mathbf{S}$ and $\mathbf{L}$ is essentially an enhancement of amplitude. This is confirmed by the harmonic dials in Fig. 8 showing the long-term solar cycle contribution to $\mathbf{S}$ and $\mathbf{L}$.

As a matter of fact we will now follow the same procedure as outlined in Section 3, and estimate the seasonal change of the Wolf ratios from the output of the modulation model by the procedure described in Section 3. The intercepts, $A$, slopes, $B$, and Wolf ratios, $M$, are compared in Table 7. As could be expected the overall values of $10^{4} M$ for the amplitude of $\mathbf{S}_{n}$ are found to be of the order of 33. Hence, the sunspot cycle enhances the amplitudes of the solar harmonics in the same proportion. The value of $10^{4} \mathrm{M}$ for the ampli- 
Table 7. Declination-Modulation model. Regression coefficients of the solar cycle effect.

\begin{tabular}{rrrrrrrrrrrrrr}
\hline & \multicolumn{3}{c}{ all days } & \multicolumn{3}{c}{$j$-months } & \multicolumn{3}{c}{$e$-months } & \multicolumn{3}{c}{$d$-months } \\
& \multicolumn{1}{c}{$A$} & $10^{4} B$ & $10^{4} M$ & \multicolumn{1}{c}{$A$} & $10^{4} B$ & $10^{4} M$ & $A$ & $10^{4} B$ & $10^{4} M$ & $A$ & $10^{4} B$ & $10^{4} M$ \\
\hline$s_{1}$ & $2.14^{\prime}$ & 69.4 & 32 & $3.07^{\prime}$ & 71.1 & 23 & $2.28^{\prime}$ & 70.5 & 31 & $1.22^{\prime}$ & 74.9 & 61 \\
$s_{2}$ & $1.45^{\prime}$ & 46.5 & 32 & $2.07^{\prime}$ & 49.3 & 24 & $1.68^{\prime}$ & 48.0 & 29 & $0.75^{\prime}$ & 51.7 & 69 \\
$s_{3}$ & $0.61^{\prime}$ & 26.0 & 42 & $0.68^{\prime}$ & 25.8 & 38 & $0.91^{\prime}$ & 25.4 & 28 & $0.25^{\prime}$ & 27.2 & 109 \\
$s_{4}$ & $0.23^{\prime}$ & 5.7 & 25 & $0.13^{\prime}$ & 3.9 & 31 & $0.39^{\prime}$ & 6.6 & 17 & $0.28^{\prime}$ & 7.0 & 25 \\
$\ell_{1}$ & $0.06^{\prime}$ & 1.6 & 26 & $0.15^{\prime}$ & 3.4 & 23 & $0.06^{\prime}$ & 3.0 & 49 & $0.04^{\prime}$ & 3.4 & 93 \\
$\ell_{2}$ & $0.13^{\prime}$ & 4.5 & 34 & $0.25^{\prime}$ & 5.6 & 22 & $0.13^{\prime}$ & 5.1 & 40 & $0.04^{\prime}$ & 5.7 & 158 \\
$\ell_{3}$ & $0.05^{\prime}$ & 1.0 & 19 & $0.10^{\prime}$ & 2.2 & 23 & $0.05^{\prime}$ & 1.6 & 32 & $0.01^{\prime}$ & 0.3 & 33 \\
$\ell_{4}$ & $0.01^{\prime}$ & 0.4 & 41 & $0.01^{\prime}$ & 0.6 & 51 & $0.02^{\prime}$ & 0.5 & 28 & $0.01^{\prime}$ & 0.5 & 80 \\
$\sigma_{1}$ & $62^{\circ}$ & -788 & -12.6 & $50^{\circ}$ & -910 & -18.2 & $66^{\circ}$ & -881 & -13.3 & $87^{\circ}$ & -945 & -10.8 \\
$\sigma_{2}$ & $233^{\circ}$ & -378 & -1.6 & $246^{\circ}$ & -204 & -0.8 & $231^{\circ}$ & -229 & -1.0 & $202^{\circ}$ & -275 & -1.4 \\
$\sigma_{3}$ & $79^{\circ}$ & -173 & -2.2 & $88^{\circ}$ & -185 & -2.1 & $74^{\circ}$ & -174 & -2.4 & $77^{\circ}$ & -157 & -2.0 \\
$\sigma_{4}$ & $280^{\circ}$ & -835 & -3.0 & $307^{\circ}$ & -491 & -1.6 & $283^{\circ}$ & -574 & -2.0 & $273^{\circ}$ & -652 & -2.4 \\
$\lambda_{1}$ & $155^{\circ}$ & 594 & 3.8 & $131^{\circ}$ & -168 & -1.3 & $180^{\circ}$ & 72 & 0.4 & $265^{\circ}$ & -77 & -0.3 \\
$\lambda_{2}$ & $283^{\circ}$ & -892 & -3.1 & $291^{\circ}$ & -469 & -1.6 & $283^{\circ}$ & -524 & -1.9 & $230^{\circ}$ & -895 & -3.9 \\
$\lambda_{3}$ & $134^{\circ}$ & 149 & 1.1 & $136^{\circ}$ & -349 & -2.6 & $134^{\circ}$ & -117 & -0.9 & $107^{\circ}$ & 789 & 7.4 \\
$\lambda_{4}$ & $303^{\circ}$ & -488 & -1.6 & $247^{\circ}$ & -653 & -2.6 & $344^{\circ}$ & -180 & -0.5 & $283^{\circ}$ & 696 & 2.5 \\
\hline
\end{tabular}

tude of $\mathbf{L}_{n}$ is somewhat smaller. Because of the coincidence of these figures with the corresponding value of 0.0033 for the Wolf ratio $M$ of the electron density in the ionospheric E-region (Malin et al., 1975) it is traditionally accepted that the overhead $\mathbf{S}_{\mathrm{q}}$ and $\mathbf{L}$ current systems are located in the Eregion. In this respect the solar cycle effect on the amplitudes of the $\mathbf{S}$ and $\mathbf{L}$ variations can be entirely attributed to changes of the E-layer conductivity.

Also very striking is the change of the Wolf ratio during the year in Table 7. The maximum of $M$ for $s_{1}$ and $s_{2}$ occurs during the $d$-season, when $M$ exceeds the $j$-value by a factor of more than 2. A similar effect is present in the $M$ values for $\ell_{1}$ and $\ell_{2}$, the Wolf ratio being about three times greater in winter than in summer. Since the Wolf ratio for E-region electron density is found to be remarkably constant in the course of the year (Malin et al., 1975), the solar cycle effect in winter is larger than can be explained by changes in ionospheric conductivity alone.

Moreover, the lunar tidal movements in the ionosphere are gravitationally induced and are therefore not expected to vary appreciably with sunspot number, so it is reasonable to assume the change of $\mathbf{L}$ with solar cycle to result purely from changes in ionospheric conductivity with $R$. Thus we might expect $10^{4} M$ to be of the order of 33 for $\ell_{1}$ and $\ell_{2}$. But the slopes, $B$, of the amplitudes of $\mathbf{S}$ and $\mathbf{L}$ in Table 7 are essentially the same for the three Lloyd seasons, whereas the intercepts, $A$, change considerably through the year. This seasonal variation of the intercepts seems to be the reason for the different estimates of the Wolf ratios $M$ obtained by the ratio $B / A$ in Eq. (3). The same conclusion can be reached for the phases of the $\mathbf{S}$ variation but for the phases of $\mathbf{L}$ the outcome is indistinct.

The observed lunar daily variation, $\mathbf{L}$, contains contributions from the ionospheric dynamo, and also from an ocean dynamo powered by the tidal motion of the sea across the
Earth's main magnetic field. Because the modulation model incorporates four harmonics of $\mathbf{L}$ we can apply the method of Malin (1970) for the separation of the contributions from the two dynamos. The amplitude, $\ell_{\mathrm{O}}$, and phase, $\lambda_{\mathrm{O}}$, of the ocean dynamo variation $\mathbf{L}_{\mathrm{O}}=\ell_{\mathrm{O}} \sin \left(2 \tau+\lambda_{\mathrm{O}}\right)$ are computed from

$$
\begin{aligned}
& \ell_{\mathrm{O}} \cos \lambda_{\mathrm{O}}=\sum_{n=1}^{4} \ell_{n} \cos \lambda_{n}, \\
& \ell_{\mathrm{O}} \sin \lambda_{\mathrm{O}}=\sum_{n=1}^{4} \ell_{n} \sin \lambda_{n} .
\end{aligned}
$$

The ionospheric dynamo part, $\mathbf{L}_{\mathrm{I}}$, of the $\mathbf{L}_{2}$ variation is described by $\mathbf{L}_{\mathrm{I}}=\ell_{\mathrm{I}} \sin \left(2 \tau+\lambda_{\mathrm{I}}\right)$, where the amplitude, $\ell_{\mathrm{I}}$, and the phase, $\lambda_{\mathrm{I}}$, are obtained by

$$
\begin{aligned}
& \ell_{\mathrm{I}} \cos \lambda_{\mathrm{I}}=-\left(\ell_{1} \cos \lambda_{1}+\ell_{3} \cos \lambda_{3}+\ell_{4} \cos \lambda_{4}\right), \\
& \ell_{\mathrm{I}} \sin \lambda_{\mathrm{I}}=-\left(\ell_{1} \sin \lambda_{1}+\ell_{3} \sin \lambda_{3}+\ell_{4} \sin \lambda_{4}\right)
\end{aligned}
$$

Thus, the ionospheric dynamo part of $\mathbf{L}$ consists of the contribution of the terms $\mathbf{L}_{1}, \mathbf{L}_{\mathrm{I}}, \mathbf{L}_{3}$ and $\mathbf{L}_{4}$. Using the results of the Chapman-Miller analysis in Table 1 it is found that the amplitude $\ell_{\mathrm{O}}=0.123^{\prime} \pm 0.015^{\prime}$ of the ocean dynamo effect, $\mathbf{L}_{\mathrm{O}}$, is well determined at the inland station of Dourbes at about $180 \mathrm{~km}$ from the North Sea and is comparable to the amplitude $\ell_{\mathrm{I}}=0.137^{\prime} \pm 0.014^{\prime}$ of the ionospheric dynamo part, $\mathbf{L}_{\mathrm{I}}$. So there is a relatively important ocean dynamo contribution to the total lunar daily variation at the station and this effect should be removed before studying time variations of $\mathbf{L}$.

It is interesting to see how this constant amplitude constituent, $\mathbf{L}_{\mathrm{O}}$, in the measured amplitude of $\mathbf{L}_{2}$ could affect the Wolf ratios $M_{n}^{\ell}$ and $M_{n}^{\lambda}$ when the influence of the nighttime tide is eliminated by subtracting the single harmonic term $\ell_{\mathrm{O}} \sin \left(2 \tau+\lambda_{\mathrm{O}}\right)$ from the original observations. As 
Table 8. Declination-Modulation model lunar variation. Removal of ocean dynamo contribution.

\begin{tabular}{ccccc}
\hline$n$ & $\ell_{n, \mathrm{o}}$ & $\lambda_{n, \mathrm{o}}$ & $10^{4} M_{n}^{\ell}$ & $10^{4} M_{n}^{\lambda}$ \\
\hline 1 & $0.1206^{\prime} \pm 0.0006^{\prime}$ & $195.501^{\circ} \pm 1.037^{\circ}$ & $22.98 \pm 0.26$ & $-0.84 \pm 0.01$ \\
2 & $0.1357^{\prime} \pm 0.0011^{\prime}$ & $293.766^{\circ} \pm 1.101^{\circ}$ & $27.75 \pm 0.28$ & $-1.88 \pm 0.02$ \\
3 & $0.0668^{\prime} \pm 0.0005^{\prime}$ & $175.509^{\circ} \pm 0.919^{\circ}$ & $28.68 \pm 0.28$ & $-1.92 \pm 0.02$ \\
4 & $0.0217^{\prime} \pm 0.0002^{\prime}$ & $304.383^{\circ} \pm 2.201^{\circ}$ & $38.36 \pm 0.25$ & $-1.03 \pm 0.01$ \\
\hline
\end{tabular}

expected, it is found that the amplitudes, phases, and Wolf ratios for the solar variation $S(t)$ in Eq. (4), with the ocean dynamo contribution removed, are practically unchanged as compared with the results in Tables 3 and 5. The principal outcomes of the modulation model are summarized in Table 8 and concern only the amplitudes, $\ell_{n, 0}$, and the phases, $\lambda_{n, 0}$, of the lunar variation $L(t)$ in Eq. (5), which is now mainly of ionospheric origin. These values should be compared with the corresponding ones in Table 1, resulting from the Chapman-Miller analysis.

After elimination of the ocean dynamo effect, the results for $c_{n, k}^{\ell}, \varphi_{n, k}^{\ell}, c_{n, k}^{\lambda}$ and $\varphi_{n, k}^{\lambda}$ are found to be nearly the same as those given in Table 4, which implies that the seasonal variation parts in the second terms in the right-hand side of Eqs. (8) and (9) are virtually unchanged by removal of the ocean dynamo contribution from the observations. From Table 8 it follows that only the mean amplitude, $\ell_{2,0}$, of the dominant $\mathbf{L}_{2}$ harmonic is significantly changed. The values of $10^{4} M_{n}^{\ell}$ for the sunspot number influence on the amplitude of the $\mathbf{L}$ variation are somewhat reduced with respect to the values given in Table 5 and are now of the order of the magnitude of the Wolf ratios $10^{4} M_{n}^{s}$ for the solar variation, whereas the factors $10^{4} M_{n}^{\lambda}$ remain small. So it is concluded that the ionospheric part of $\mathbf{L}$ comprehends almost the same Wolf ratios as $\mathbf{S}$. The Wolf ratios for both $\mathbf{L}$ and $\mathbf{S}$ are of the same order of magnitude for all harmonic numbers $n$, which means that the Wolf ratios are nearly independent of frequency.

\section{Conclusions}

A spectral analysis is performed to determine the components of the solar and lunar variations present in the Dourbes magnetic declination record, data for a period of 40 years (1960-1999) being used. The amplitude spectrum shows prominent solar peaks at 1, 2, 3, 4 cycles/day (cpd), and lunar peaks at $0.93227 \mathrm{cpd}$ and $1.93227 \mathrm{cpd}$. The high resolution spectral analysis also displays splitting of the solar diurnal and semi-diurnal lines, $\mathbf{S}_{1}$ and $\mathbf{S}_{2}$, by the annual variation and its harmonics, and an annual splitting of the lunar semidiurnal line, $\mathbf{L}_{2}$. Sidebands of the solar diurnal peak $\mathbf{S}_{1}$ at $(1 \pm k / 27) \mathrm{cpd}, k=1,2,3$, are attributed to an amplitude modulation mechanism related to solar rotation.

The extra peaks in the solar frequency bands are interpreted in terms of amplitude and phase modulation of the main dynamo constituents by the annual variation of ionospheric conductivity. As a generalization of the harmonic model used in the Chapman-Miller analysis it is possible to describe the solar and lunar geomagnetic variations in continuous time as phenomena of the modulation type and thus give up the idea of periodic behaviour that is resolved into a discrete set of amplitudes and phases of pure harmonic waves. The fundamental concept of the modulation model consists of the sum of harmonic oscillators with basic carriers having the primary frequencies of the solar and lunar daily variations, which are subjected to amplitude and phase variations that can be represented by a sum of trigonometric functions with frequencies given by the harmonics of the annual variation. The effect of changes in solar activity is explicitly taken into account by incorporating the daily sunspot numbers as an additional input into the modulation model.

The solar and lunar daily variations exhibit important seasonal amplitude and phase modulations. When the data are examined month by month, some striking changes are found in the solar magnetic variation resulting from thermal tides and also in the lunar tides, involving an exceptional phase shift between local winter and summer with rapid changes in the equinoctial months. From the point of view of amplitude amplification, the enhancement of $\mathbf{L}$ from winter months to summer months significantly exceeds that of $\mathbf{S}$. The phase change in the lunar current system between local winter and summer is more than twice that of the $\mathbf{S}$ system.

The long-standing question of whether $\mathbf{S}$ and $\mathbf{L}$ respond similarly to the sunspot cycle is re-examined using 40 years of data and ranging over nearly four solar cycles. Therefore, intermediate values of $R$ between sunspot minimum and sunspot maximum are included so that the outcomes may be interpreted to be fairly representative of the average solar cycle influence on $\mathbf{S}$ and $\mathbf{L}$. The sunspot cycle influence on the amplitude and phase of the solar and lunar variations is estimated a priori by incorporating the Wolf ratios in the modulation model as additional regression parameters. The Wolf ratios for the amplitude of the $\mathbf{S}$ and $\mathbf{L}$ variations are found to be nearly the same, but no systematic changes in the phase angles with increasing sunspot number are discernible, implying that the solar cycle effect on $\mathbf{S}$ and $\mathbf{L}$ is essentially an enhancement of amplitude. As the analysis only uses the data from one magnetic observatory, no affirmative statement concerning the possible different response of $\mathbf{S}$ and $\mathbf{L}$ to sunspot number can be made.

The amplitude of the ocean dynamo contribution to the lunar daily variation is relatively large in the magnetic declination data for a station at a distance of about $180 \mathrm{~km}$ of the coast, and is comparable to the ionospheric dynamo amplitude. Lunar Wolf ratios for the sunspot cycle influence are of the order of the Wolf ratios associated with the solar variation when the ocean dynamo effect is separated from the lunar daily variation. Then the lunar Wolf ratios are reduced by about 10 per cent.

Acknowledgments. Special thanks are due to G. Simon of the 
observatory of Dourbes for producing a virtually gapless record of high quality hourly magnetic data over the past 40 years. The author is very indebted to the referees for useful comments.

\section{References}

Black, D. I., Lunar and solar magnetic observations at Abinger: Their detection and estimation by spectral analysis via Fourier transforms, Phil. Trans. R. Soc., 268 A, 233-263, 1970.

Broyden, H., Quasi-Newton methods and their application to function minimisation, Math. Comp., 21, 97-105, 1967.

Campbell, W. H., The regular geomagnetic-field variations during quiet solar conditions, in Geomagnetism, vol. 3, edited by J. A. Jacobs, 76 pp., Academic Press, New York, 1987.

Chapman, S. and S. R. C. Malin, Atmospheric tides, thermal and gravitational: nomenclature, notation and new results, Journ. Atmos. Sci., 27 707-710, 1970.

Chapman, S. and J. C. P. Miller, The statistical determination of lunar daily variations in geomagnetic and meteorological elements, Mon. Notices Roy. Astron. Soc., Geophys. Suppl., 4, 649-669, 1940.

Chapman, S., J. C. Gupta, and S. R. C. Malin, The sunspot cycle influence on the solar and lunar daily geomagnetic variations, Proc. R. Soc. London, A 324, 1-15, 1971.

Coulson, C. A., Waves, 159 pp., Oliver and Boyd, Edinburgh, 1955.

De Meyer, F., Modulation of the solar daily geomagnetic variation, J. Atmosph. Terr. Phys., 48, 115-130, 1986.

De Meyer, F., Modulation of the solar magnetic cycle, Sol. Phys., 181, 201219,1998
Green, P. and S. R. C. Malin, Lunar and solar daily variations of the geomagnetic field at Waterhoo, West Australia, J. Atmosph. Terr. Phys., 33, 305-318, 1971.

Malin, S. R. C., Separation of lunar daily geomagnetic variations into parts of ionospheric and oceanic origin, Geophys. J. R. astr. Soc., 21, 447-455, 1970 .

Malin, S. R. C. and S. Chapman, The determination of lunar daily geophysical variations by the Chapman-Miller method, Geophys. J. R. astr. Soc., 19, 15-35, 1970

Malin, S. R. C., A. Cecere, and A. Palumbo, The sunspot cycle influence on lunar and solar daily geomagnetic variations, Geophys. J. R. astr. Soc., 41, 115-126, 1975.

Marquardt, D. W., An algorithm for least squares estimation of nonlinear parameters, Journ. Soc. Industr. Appl. Math., 11, 431-441, 1963.

Matsushita, S., Solar quiet and lunar daily variation fields, in Physics of Geomagnetic Phenomena, edited by S. Matsushita and W. H. Campbell, pp. 302-424, Academic Press, New York, 1967.

Matsushita, S. and H. Maeda, On the geomagnetic solar quiet daily variation field during the I. G. Y., J. Geophys. Res., 70, 2535-2558, 1965.

Tarantola, A. and B. Valette, Generalized nonlinear inverse problems solved using the least squares criterion, Rev. Geophys. \& Space Physics, 20 219-232, 1982.

Winch, D. E. and R. A. Cunningham, Lunar magnetic tides at Watheroo: seasonal, elliptic, evectional, variational and nodal components, J. Geomag. Geoelectr., 24, 381-414, 1972.

F. De Meyer (e-mail: Frans.DeMeyer@oma.be) 